Anger.

De Papyro fuctice, won der papierstande ad Esaiae $X \mid X, 7$ 


\section{$195+2$}

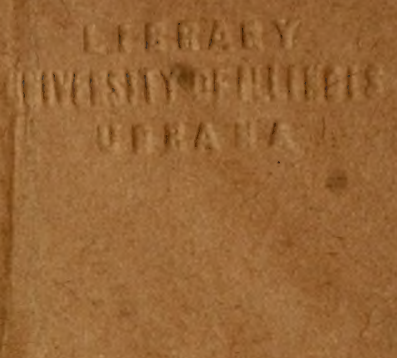




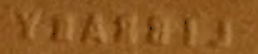

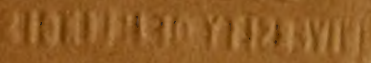
Ansesso
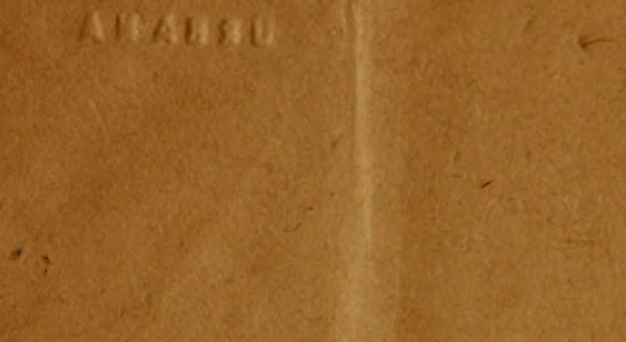


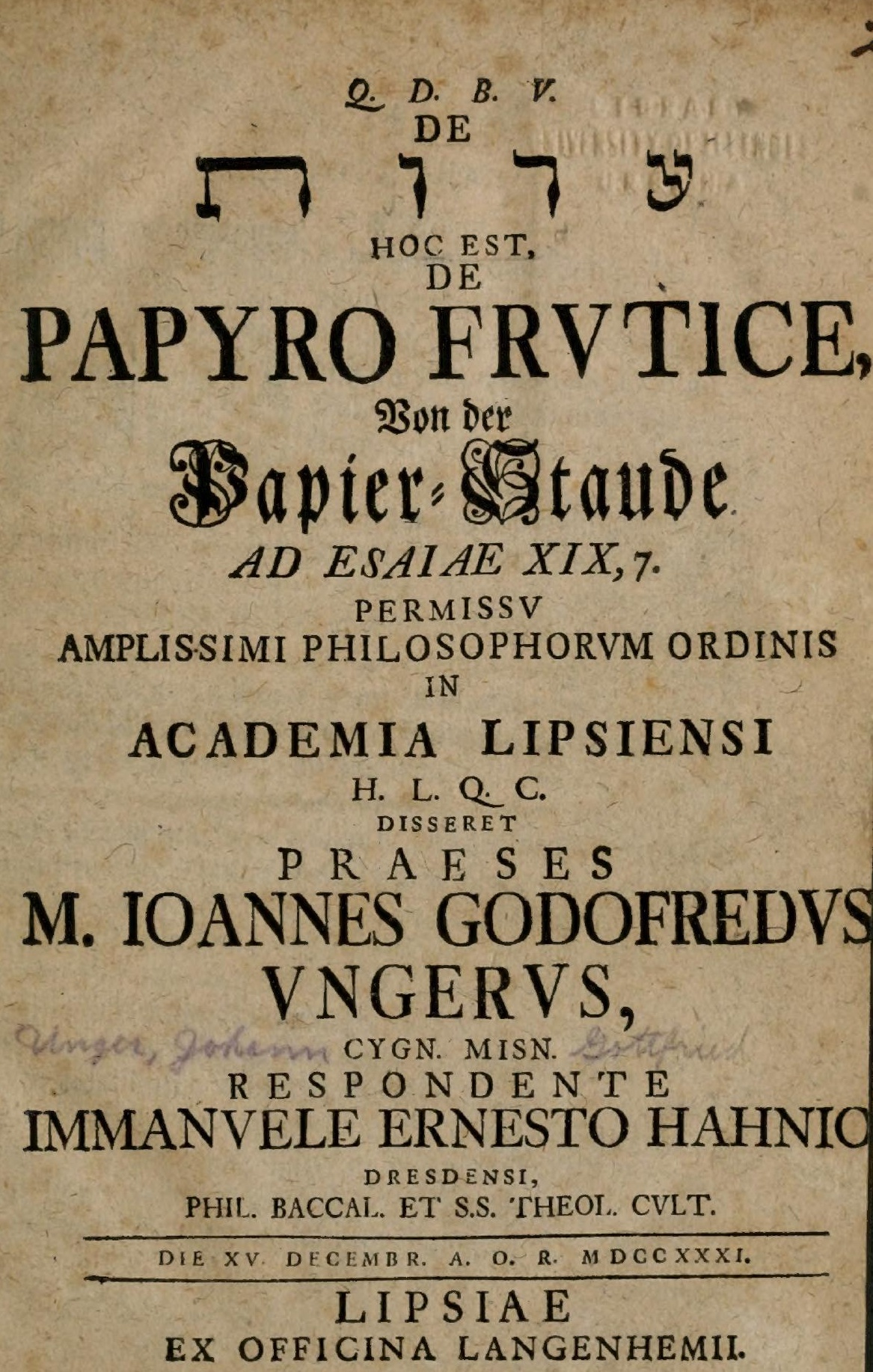




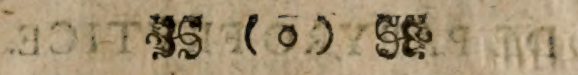 \\ IEHOVA IVIKA!}

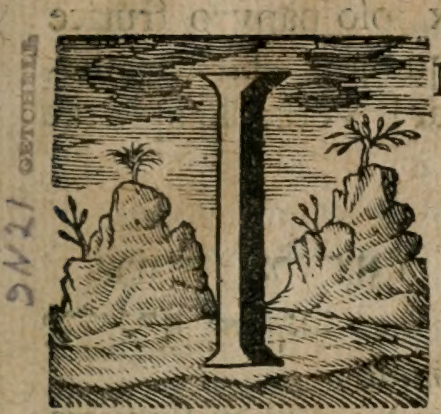

\section{Q. I.}

Nter fümma, quae Benigniffimum Numen hominibus contulit, bona papyrum effe referendum, qui inficias eat, neminem fore arbitifor. Siue enim quis vtilitatem fequatur, vix quidquam vtilius,, ue excellentiam, vix quidquam praeftantius, fiue aptitudinem fenfa animi alis communicandi, vix quidquam aptius in rerum natura effe deprehendet. Vnde fententiam CAII P LINII MAIORIS *) merito collaudamus, qua, cbartae, inquit, vfu maxime bumanitas vitge conftat et memcria. Ad quod quidem effatum verifimum MELCHIOR GVILANDINVS, **) felix horum verborum interpres, praeclare ait: Dixit Plinius chartae vfu maxime buN.manitatem vitae conflare et memoriam, propter libros, in quos praecepta bene viuendi, res geftas, artes pluriQ mas, praeclaras animi noftri meditationes, et multa alia, nomine nunquam interituro, immortaliterque defcribi-

ণ mus, ac praefertim propter officium, quod abfentibus, Z etlongo a nobis interuallo diffitis, per literas praeftamus. \$.II.Cum autem charta, qua vtimur, hodierna a papyro, Aegypti frutice praeftantifimo, nomen fuerit fortita, et dulciflimus vates Efaias diuinum de eo nobis refliquerit oraculum, explicatione longe dignifimum; hine * nos, antequam aliud, quod in Pfalmos Dauidis meditaC mur Opus emitteremus, de eo differere non noluimus. Faxit modo Numen Optumum Maxumum, vt vel hocce A 2 inft-

*) L. XIII. c. XI. Hiftor. Natur.

*) Membr. I. Comment. fui in tria C. Plinii Maioris de papyro Capita p. 28.Edit.Amberg.1713. 


\section{DE PAPYRO FRVTICE.}

inftitutum noftrum in eius vergat honorem; vt eius nomen, quod in regno naturae ex ipfo papyro frutice eminentiffime emicat, ab impiis quoque vlterius inueftigetur pariter atque amplificetur! Verba Prophetae ita fluunt :$$
\text { Ief. } X I X, 7 \text {. }
$$

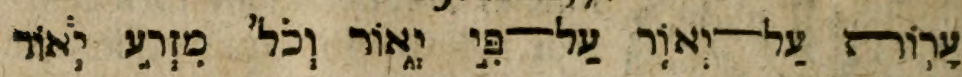
Verfio Sebaft. Scbmidii: :

Papyri iuxta flumen (Nilum), iuxta os fluminis, et omne femen fluminis exarefcet, impelletur, et non erit (amplius).

\section{Reformata German.}

Desgleidjen bie Papyr: Stauben an Den ßádjen,

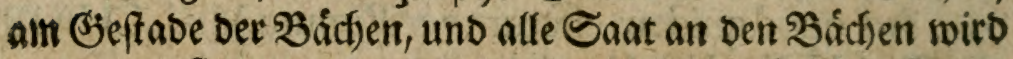
berDorten, fie wirb vertrieben werden, und nicht mefor fenn.

Belgica.

Het Papier-Gewas by de Stroomen gen de Oevers der Stroomen, ende al het Gezaeyde aen de Stroomen, fal verdroogen, het fal wechgeftooten worden, ende niet (meer) zijn.

\section{§. III.}

Quilibet, me non monente, intelligit, in hoc oraculo de 5 in h. e. de papyro, frutice Aegypti famigeratiffimo, fermonem effe. Et cum varia de fignificatu, natura, hiftoria ac fine Creatoris circa 5 ער veniant ponderanda, ea autem, quae alii de hac materia fcripferunt, aut non fufficiant, aut obfcure admodum et confufe fint tradita; hinc nos noftrarum partium fatacturos effe exiftimauimus, fi quatuor Capitibus haec omnia figillatim exponeremus, Lectorique adeo decenti ordine lucem affunderemus. Infcripfimus hanc ob caufam 


\section{CAPV'T I.}

D E

\section{CONSIDERATIONE PHILOLOGICA עיר SEV PAPYRI.$$
\Sigma \Upsilon N O \Psi \text { I } \Sigma \text {. }
$$

Connexio vaticinii buins cum Diffentientes re nobifcum conpraecedentibus S.I.

Cum confegq. S.II.

Obiectum f. คำ in quo cardo totius rei vertitur. S. III.

Difficile, vtile tamen eft banc vocem inquirere. S.IV.

Diuortia Jententiarum circa Explicantur reliquaverba bueius Jignificatum $\mathfrak{g} . V$.

Notat veropapyrum, quod probatur

a. ab origine vocis, defcendit enim ab רาบูง.VI. non aeque $a b$ רiע contra Kimchium f. VII.

2.ab emphatico pofitu verborum

Prophetae S.VIII.

3.ex bifturia Nili $\mathcal{S} . I X$. veniunt $\$$. XII.

910 et 1 dunt Jynomyma ชชี โาำ ขednotant papyrum communem $\$$. XIII.

$L X X$. viralis interpretatio refutatur $\$ . X I V$. Inde ius verficulis. $X V$.

בהואר Euphratem,quod obferuatur ib.

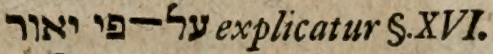
Per

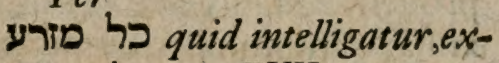
ponitur S. XVII.

Uב proprie accipitur \$.XVIII. ๆา emplatice ponitur, quod $\operatorname{docet} \$$. $X I X$.

quod papyrus etiam in Sy- Senfus verficulinofriproprius ria, et ad Euphratem gi- eft $\$ X X$. Et $\sqrt{2 i c}$

gnatur, buc non pertinet S.X. Tranjitur ad Caput fecundum 4. ex confenfu Ebraeorum S.XI. S. XXI.

S. I.

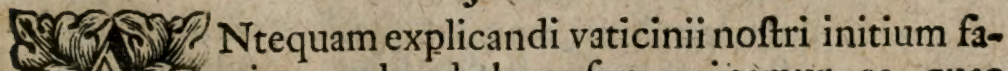

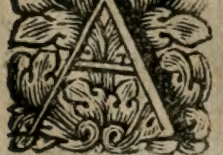
ciamus, haud abs re fore opinamur, ea, quae antecedunt pariter ac quae fequuntur praelibare. Praedixerat hactenus Cap. XVIII. vates Aethiopibus fata feralia ; iam totus eft in eo, vt Aegypto quoque, regno tum temporis florentifimo, de- 
crementa et ruinam den unciet. Idque vt ad oculum quafi demonftret Aegyptiis, nil tale metuentibus aut fperantibus, fingulas res, internecionifuccubituras, prolixe in hoc Cap. XIX. enarrat. Quam narrationem exorditur a deftruendis idolis, feu religione Aegyptiorum v. r-3. Deinceps pergit ad deftructionem regni ac Principis. Aegyptiorum, quippe non amplius fub aulpiciis pacifici aut clementis Regis, fed fub manu duri domini vitam effent transacturi v. 4. Hanc calamitatem excipit inopia victus et amictus, omnes cnim fluuii, omnes amnes, omnes aluei cum prouentibus fuis exficcandi praedicuntur v.5. Prouentus autem illi fluminum, quorum defectus dicitur exfpectandus, ftatim v. 6. recenfentur, nimirum gा0) ptiorum, vnde maxima pars alimentorum proueniebat, marcefcent. Cum itaque fpeciatim horum fecerit mentionem Propheta, neque 5. II.

Haec funt, quae antecedunt oraculum noftrum. Stupenda vero díxeris ea, quae idem infequuntur. Nempe ex prouentibus illis fluuiorum, inprimis $\mathrm{Nili}$, dependebat maXima, tantum non omnis copia et vbertas Aegyptiorum. Quare av: 8-rr. lacrymae et planctus pifcatorum defcribuntur, fiquidem exficcatis fluminibús occafio hamum in mare

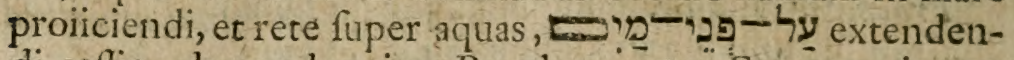
di ceflat, de quo loquitur Propheta v. 8 . Et vt paucis verbis, neruofe tamen ac ponderole, pro more fuo, rem expri-

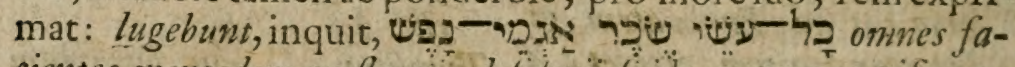
cientes mercedem ex flagnis defiderii Jui h. e. omnes pifcatores V. IO.

\section{III.}

De antecedentibus itaque ac confequentibus cum inter nos conftet; ante omnin Obiectum, quod eft papyrus, erit inuefigandum. Quippe in papyro defcribendo nos operam noftram qualemcunque effe collocaturos promifimus, papyrus igitur veramque facit paginam fermonis. Cum vero vox illa รin in fronte verficuli noftri pofita, tum 


\section{DE PAPYRO FRVTICE.}

aliis, tŭm nobis nomine papyri veniat, idque optimo iure; hine cardinem rei in inquirendo, enodando, ftabiliendo huius vocabuli fignificatu verti exiftimamus.

\section{IV.}

Negotium autem eft certe periculi aeque ac laboris pleniffimum rem hanc conficere. Requiritur enim, vt quis hiftoriae A gegptiorum apprime fit gnarus, et in promptu habeat fruticum prope ripam et vndas Nili nafcentium naturam, viumque, vt videlicet omnibus praefidiis ex antiquitate ftudiofe collectis folidi aliquid et veri,imo certi de papyro concludi polfit. Neque putandum eft, laborem, qui rebus huiuscemodi inueftigandis et perquirendis impenditar, inutilem effe planeque fterilem. Quo altius enim omnis diffutatio arceffitur, hoc iucundior et vilior effe debet, vel ideo, quia vna idemque opera illuftris Scripturae diuinioris locus explanatur.

$$
\text { S. V. }
$$

Quid vox ᄀบ צִ fignificet magna funt fententiarum diuortia. Alii enim vertunt: nudabitur, in quibus HIERONYMVS, a)nec non THOMAS AQVINAS, b) Doctor ille Angelicus, quos tamen errare in forma verbi vel lufcus videt. Alii $\alpha^{\prime} \chi^{i}$ h. e: vluam, caricem reddunt, quod fecit LXXviralis interpres, c)quem THEODORET. d) et CYRILLVS ALEXANDRINVS aliique ex Patribus fequuntur. Alii tranftulerunt grannina, e) ad quos B. MEGALANDER, 1O. OECOLAMPADIVS et GALLICVS interpres pertinent. Alii germina, iuncum, foirpum hoc nomine exprimi volunt, in quibus 10 . COCCEIVS, VRSINVS, SALMASIVS.f) Alii denique, et quidem plerique, quod infra docebimus, papyrum interpretantur. Nos equidem neminem tantorum S. Codicis interpretum laude fua defraudamus; interea tamen fignificatum vocis huius effe papyrum, eorum pace defendemus.

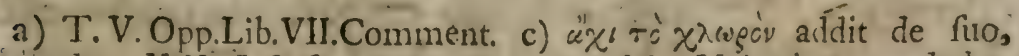
ad c. XIX. Ief. f. 4 I.

b) T.XIII.Commentar. f.2I.Edit, Antwerp. quod in Hebraico non habetur, inquit HIERONYMV S l.c.

$$
\text { c) T.II. }
$$




\section{DE PAPYRO FRVTICE.}

d) T. II. Opp. ad c.XIX.Ief.f.72. Edit.Parif. 1642. De CYRILLO ALEXANDR. infra erit locus dicendi.

e) B.LVTHERVS expreffe: ธas Srafi reddidit. IO. OECO. LAMPADIVS in Comment. in Prophetas ad c. XIX. Ief. f. 136. vertit gramina. Quibus cum Gallica Verfio: les prairies confentit. $\Lambda \mathrm{b}$ his non abludunt ii, qui gramineta, olera, cum PAGNINO ac MERCERO tranfponunt, quos refutat MATTHIAS HILLERVS in Hierophyt. P. II. c. 38. p. 313. fq. vocem, inquit, mo qui gramineta vel olera verterunt, Synonimon eam putarunt nominis nורי; fed lapje funt per errorem, quia y littera licet gutturatis annimeretur, cum $\times$ nun- quam permutatur, caque ds cauja eiusdem Jignificatus vo: cabulum non conftituit.

f) IO. COCCEIVS in ' T.II. Opp. Comment.inlef. f.285.germina fupra amnem vertit, quod idem facit in Lexc. C Coinmentario Sermonis Ebr. et Chald. p.645. VRSINVS in Arboreto biblico p. 158. חาע papyrum notare vltro quidem fatetur, red iuncum, fcirpum sagis idein cum co effe vult. Nec longe abeft SAL.MASIVS in Exercitatt. Plinian. p. 7\%5 qui equidem Hebraicum vocabulum non habet, $\phi \lambda \tilde{\varepsilon} \nu$ h. e. feirpum tamen papyri Synonimon effe docet, ea de caufa, quoniam fimilitudinem et vfum Niloticae babuerit plantae,quod tamen erat probandum.

\section{VI.}

Quantumuis autem vox haec fit $\alpha^{\prime} \pi \alpha \xi \lambda \varepsilon \gamma^{\prime} \mu \varepsilon v o s$ in S. Codice, et tam multifariae interpretum opiniones; nos tamen haud inualidum argumentum, pro euincendo fignificatu papyri, ab origine vocis peti poffe, nobis perfuafum habemus. Defcendit autem vox radix apud Hebraeos quatuor potifimum obtinet fignificationes, prima eft euacuari, effundi, quafi effet a Graeco d'sesv vel cugesv tollere. a) Altera eft nudare, retegere, difcooperire.b) Tertia eft adbaerere, cubaerere, $c$ ) et quarta denique eft virefcere, quia viriditas arboris vel plantae alicuius integritatem indicat, fuccique in ramos et folia virentia largam effufionem importat. d) Quam vltimam verbi ער מר notationem facimus noftram, praefertim cum in ea opinione

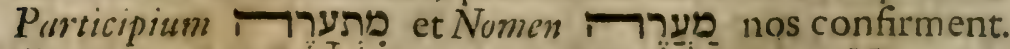
Illud cum praefixo i legitur Pf. XXXVII , 35. Hoc cum praefixo D lud. XX, 33. lllud notat omne virens hoc locum? 
berlis virefentibus abundantem. Vtrumque eft a radice กา. Quibus cum fi componas $\bigsqcup_{\text {, }}$, habebis virentia h. e. papyros, frutices virentes.

a) Vti AVENARIVS loquirur in Libro Radicum p. 599. Hanc autem enacuandi fignificationem habet ערה in Piel. Vnde in Fut. Piel in 2. $\int$ mafc. . per Apocopen autein 3. rad. 3 legitur רघูnฺ effundes Pr.CXLI,8. Sic in eadem forma Apocopata

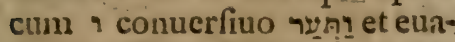
cuauit. Gen. XXIV, 20. add. Cliron. XXIV, Ir.

b) Hoc fignificatu notum eft Perf. Piel ער retexit, Zeph. II, 14. Infinit. Piel niny retegendo Hab. III, I2. Fut. in Piel העי"? nudabit Ef. 1II, 17. Vnde natales traxit vox עריה retectio Mich. I, II. Ezech.XVI, 7. Alia nomina eiusdem fignificatus, luc pertinentia, operofe et laudabiliter collegit ROBERTSON in Thefaur. Lingu. $S$. p. $77 \mathrm{r}$.

c) Quod pluribus ex Rabbinis oftendit BVXTORFFIVS in Lex. Chald. Thalnuud. Rabbin. f. 1664 .

d) Vti egregie obferuauit STO. CKIVS in Claue Lingu. S. p. in. 813 .

\section{VII.}

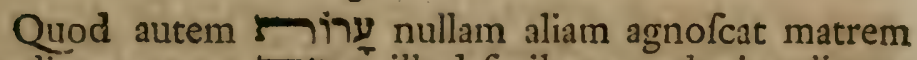
feu radicem, quam $\rightarrow$ ר. rum nominum, eodem inodo a verbis ${ }^{\prime \prime} \zeta$ formatorum colligere eft. Sic habemus a radice $\square$ nomen plurale

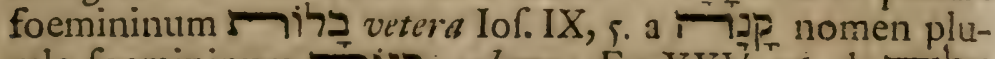

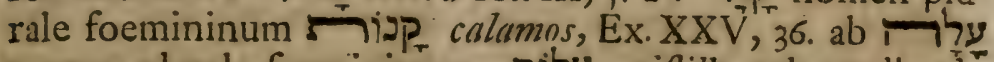
nomen plurale foemininum תity piftilla, ab attollendó fic dicta 2. Chron. XXIV, I4. Et PAGNINVS ait: Nominatiuus pluralis foeminini bi רy. a) Facili autem negotio refutari poteft R. D. KIMCḦivS ad h.l. qui עִ nobifcum vercit quidcm papyros, fed nomen רiע quod notat cutem, pellem, adeoque radicem עatrem effe mauult, ideo, quia papyri in praetenues et multiplices membranulas acu fuerint diuifae. Nam רiע pariter $a b-7$ y defcendit, et, quod probe notandum obferuandunque eft, mutant ac mutuant; b) practerea acus plane erat inhabilis ad membranulas a papyro diuellendas, quod infia Cap. II. §. XIV. ex GVIL, A NDINO audiemus. 
a) Nos formamus תוาע ita. ער in Particip. habet עִ et hoc eft nomen generis mafculini. mutatur in gen. faeminino in habes cedimus in tribus allegatis eiusdem formae Nominibus. Denique Faemininum Singul. $\rightarrow$ in Plur. tranfit in ri iuxta regulas Grammaticorum, inde efficitur תiา b) Hoc obferuandum eft tumvt non fallamur in radice, tum ve ex tricis Lexicographorum eripiamur. Obferuationem autem hanc illuftrabimus. Sic a radice noftra הา2 eft Imperat. nาุy nida te Ief. XXXII, II. forma petita a rad. $972 \mathrm{cum} \rightarrow \mathrm{Pa}$ ragogico. Sic Fut. าโบภn nudabitur Hab. III, 9. vbi forma rurfum $a b$ ๆ eft mutuata.

\section{VIII.}

Quemadmodum autem origo vocis eiusque fignificatio fatis pro noftra fententia loquuntur; ita non minus emphatica pofitio ac cobaerentia verborum Prophetae rem confirmant. Emphatice enim exclamat: רiאי -

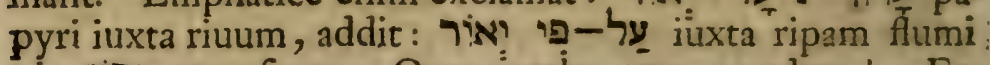
nis שב:? exarefcent. Quot verba, tot pondera! Exaggerat enim vates rem, praedicens omnibus Aegypti incolis, papyrum praeftantiffimi vfus fruticem, ex quo toc commoda ad omnes redundaffent, et, vt dicamus quod res eft, qui tom egregie viruifjet, effe exariturum, humoreque priuatum iri. a)

a) Recte hoc nobifcum vrget VITRINGA in Comment. ad Ief. p. 747. quando Verfionem LXX. viralem refutat. Verba eius ita flunnt; neutiquam-concedo, illum (Graecum interpretem) vocem ער bic recte tranfuliffe per fuum äx', cum smplatica hic maxime fit oratio Propbetae, et de boneftiore ac

proprio magis Nili prouentu loqui potuerit, quam de vlus vel carice. Ergo ne longior fin: intelligo hic papyrum, celeberrimum Aegypti fruticem, productum peculiare Nili et riuorum, ex co ductorum, atque paludum Aegypti, quas effecit Nílus.

\section{IX.}

Tertio loco fententiam noftram confirmamus ex bifturia fluminis Nili. Iam vero conftat, quod praccipuus prouentus ac frutex Nili fuerit papyrus. $A b$ omnibus enim fcriptoribus, qui faltem fruticis huius mentionem faciunt, 
quo THEOPHRASTVS, HERODOTVS, CYRILLVS ALEX. PLINIVS, SOLINVS, SALMASIVS, GVILANDINVS, MONTFAVCON aliique a) pertinent, afferitur, quod ad fcribendum, ad edendum, ad calefaciendum, ad nauigandum incomparabilis eius fuerit vfus, quod infra Cap. III. pluribus exponemus. Hiftoria itaque, haud vltimum in Exegefi dubiorum locorum adminiculum, fuadet, vt de nullo alio magis, quam de papyro frutice circa vocem ราเ cogitemus.

a) Alii ex proferfo vt PLINIVS, GVILANDINVS, et MONT. FAVCON de hac materia

fcripferunt,alii incidenter, quorum teftimonia infra 1 . c. adduceinus.

5. $X$.

Nemo hic obiiciat nobis 'THEOPHRASTI a) verba; quando, nafcitur, inquit, et in Syria papyrus, circa locum, quo et calamus odoratus : vnde et Antigonus in re nauali funes feciffe proditur; aut verba PLINII, b) qui vult, papyrum gigni quoque in Euphrate circa Babylonem; nihil enim contra nos faciet, cum totum caput XIX. ESAIAE, ex quo verficulus nofter eft excerptus, de calamitate Acgyptiis inferenda c) agat, vbi Nilus, fluuius notiflimus, non Euphrates deprehenditur. Idem refpondemus is, qui GVILANDINI, fummi viri, nobis opponant experientiam, quippe qui de papyro d) teftor, ait, me vidiffe in CHALDAEA, qua duo amnes Tigris et Eupbrates concurrunt peramplas paludes, ex quibus papyrum nihilo discrepantem a Niliaca Gennefareticaque meis manibus erui atque cuulfi. Non enim contra Euphratem, fed Nilum directum eft vatis noftri oraculum, vt patebit ex $\$$. XV. huius Cap.

a) L. IV. c. 9, de Hiftoria plan- verbi diuini contra Acgyptum tarum.

b) Verba ad c. II. 9.3 adducemus.

c) quod flatim infcriptio Capitis

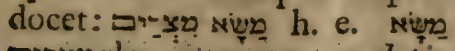
ם

conf.Malach. I, I. Zach. IX, I. d) MELCHIOR GVILANDINVS de papyro in Membr.IX. p. 276. 21. 35. Edit. Amberg. 


\section{XI.}

Denique his praemiflis recte prouocamus ad confenfum tum veterum Ebraeorum, tum recentiorum Criticorum ac Commentatorum. Iftis tefte BVXTORFFIO, a) et VRSINO,b) nota fuit vox רפי aiunt, calculum adponunt KIMCHIVS et AVENARIVS.c) Illi recentiores IVNIVM, TREMELLIVM,SCHMIDIVM cum ROBERTSONE aliisque non habent diffentientes; hi vero non folum VITRINGAM et HILLERVM, d) fed et VARENIVMe) fuos agnofcunt defenfores; ve de Verfionibus fupra allegatis et laude fua dignis nihil dicamus.

a) in Lex. Chald. 'Ihalmud. et d) MAT'TH. HILLERVS, AbRabbin. p. 1780. vbi ex Talmude Hierofolymit. Maffech. Sota cap. vlt. adducit h. e. veftes papyraceas.

b) VRSINVS in Arboreto biblico p. 158. inquit: papyri vox in.. plerisque linguis haefit. Nam et Rabbini vtuntur eo פ פ

c) KIMCHIVS ad h. l. et AVEENARIVS aliique verterunt ערות papyros Et HIERONYMVS 1. c. in notis : cum, inquit, quaererein ab criditis, quid bic. fer-

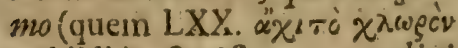
reddidit) fignificuret: audiui ab Acgyptis boc nomine lingua corum omne, quod in palude virens nafcitur, appellari.

bas ille Regiofontanus, quem tantopere laudat in Praef. SALOMON PFISTERVS, P. P. Bebenhufanus, nos, ait, (poft laudatum IVNIVM ac TREMELLIVM) tum notatione et forma vocis, tum antitbeto in altera parte buius verficuli pofito, adducimur, vt interpretemur Bißnov f papyrum, vide eun pluribus in Hierophyt. P. II. c. 38. p. 214 .

e) in Commentario fuo ad $h . I_{\text {. }}$ Facit, inquit, pro bac (fignificatione papyri) oppofitio fativarum in $m$. 2. cum papyrus infatiua fit, facit radicis applicatio. Praeclare.

\section{§. XII.}

Diffentientes plerique facillime cum noftra fententia

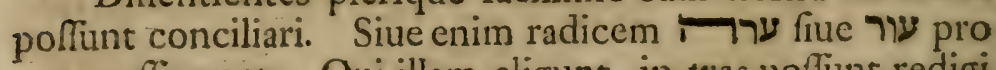
matre aflumunt. Qui illam eligunt, in tres poffunt redigi claffes: Namque aut prouocant ad fecundam fignificationem mudare, vnde HIERONYMVM et AQVINATEM in forma a) errare fupra $\$$. V. diximus; auc prouocant ad tertium fignificatum adbuerere cum OECOL $A M P A D I O$, qui granina, minutis radicibus adhaerentia Ebracis dici ait ad h.l. 
et cum. MER CERO, qui vertit herbas virides, quafi ádhaerens. tes riwis fuminibusque, et in hoc non multum, ve quilibet videt, a nobis diffentiunt; b) aut denique ad quartam nobifcum prouocant notationem, et inde papyri virent is emergit fignificatio. Qui vero hanc pofteriorem עו radicem malunt, quod KIMC.HIVS aliique c) cum eo faciunt, illi formas verborum עา ע पuas inter fe mutant faepe ac mutuant, confundunt, vti iam fupra $\$$. VII. obferuauimus.

a) Etiamfi dicere velles per Hebraifinum: mudando nudabitur; tamen non quadraret על-ליאר הeficiente Nomine, quod fubicctum exprimeret, et practerea non video, quem in finem debeant coaceruari verba: sudabitur, exare/cet, dipelletur, non erit.

b) Idem cenfemus de Verfione B. Lutheri nec non de Verfione. Gallica.

c) v. c. HILLERVS fupra Iaudatus ab עור deriuat ת quoniam in multas plagas frutexille fuerit fecatus.

\section{fi. XIII.}

Reftat adhuc magna lis circa Synonima vocis Nos laudamus eruditam obferuationem VI RI Magnifici, Patroni ac Hofpitis noftri Summe Venerabilis et incomparabilis DEYLINGII, a) obferuandum, inquit, quod $910 \mathrm{et}$

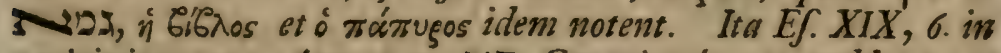
vaticinio contra Aegyptum 710 Graeci mámvgov reddunt, et Exud.II, 2. Ionutban babet 5 במי pro בתסק. Hoc tamen non obftante, adhuc netandum, quod papyrus alia fuerit communis f. vulgaris, alia Nilotica, Aegypto propria, vti peregregie monet SALMASIVS. b) Adeoque et fic rem perfacile expediemus, fi quis verficulum, Textum noftrum antecedentem cum LXX. reddat papyrum, nempe nimum ערות fed notat papyrum communem, תาע vero notat proprie papyrum, fruticem illum Niloticum, hinc vates nofter tam emphatice de eo exclamat: ערות על יאור

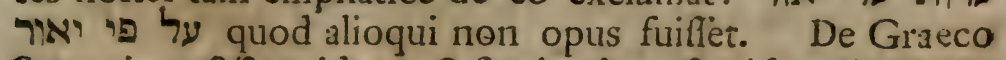
Synonimo B:B iros idem eft fentiendum, fiquidem EVSTATHIO auctore, in iis, quae ad XXI. Odyfleae rhapfodiam concinnauit, Bißnos eft planta duplex: alia papyrus Aegyptia:

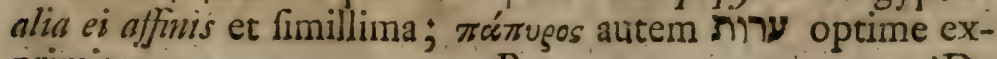
primit.

B 3

a) $\mathrm{De}$ 
a) Dedit eam in Difput. de fletu fuper 'THAMMVZ, Parti III. Obferuation. fuarum adiecta,

\section{§. XIV.}

Numerum fecus fentientium claudat interpres LXX. viralis Graecus, qui noftrum תוצ tranfulit per $\tau 0^{\circ} \alpha^{\prime \prime} x^{t}$, vluam $\mathrm{f}$ caricem.Equidem CYRILL,VS, Alexandriac Archicpifcopus a) colligit, vocem ró $\alpha^{\prime} \chi^{b}$ effe vfus Aegyptii, quod et poft eum alii ex Siracide credunt, qui $\alpha^{\prime} \chi_{b}$, inquit, b)

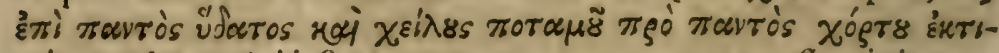
$\lambda$ nंбहrळ! h. e. Achi fuper omnem aquam et oram fluminis ante omme foenum euelletur; fcripfit autem haud dubie haec Siracides in Aegypto. Huc accedit, quod rò $\alpha^{\prime} \chi$, cenfente COCCEIO, c) non fit ab אָד alienum, quod bis legitur in Pandectis S. Gen.XLI, 2, 18 et Iob. VIII, Ir. Verum enim vero quidquid de eo fit; tamen reiicimus hanc Verfionem, cum fupra a nobis allegata argumenta eam fatis fuperque refutent, et, quod Propheta, tam ponderofis verbis vfus, non de honeftiore ac magis proprio Nili prouentu loqui voluerit, quam de carice, vix ac ne vix quidem fit probabile. Plura fi addere vellem, operam haud dubie perderem, cum pluribus ante me id fecerit VITRINGA I. c. p. 747. Idem iudicium efto de iis, qui תוาy germina, iuncum, firpum expofuere, quos vide fupra $\$$. V.

a) L. II. Tomo IV. in Ef. p. 286 . c) in Lexico fuo f. 645.

b) Sirac. C. XL, v. 16.

\section{§. $\mathrm{XV}$.}

Hoc pacto, vti cenfemus, fatis fabiliuimus ac vindicauimus fignificatum vocis צות. Peritringamus etiam leui brachio reliqua Prophetae noftri verba, quae quidem de frutice vtiliffimo Aegypti protulit. Nimirum

רוצ' quemibet riuum intelligit, vt v.antec. 6. fed in fpecie Nilum, alias מיזים 2. Reg. XXIV, 7. KIMCHIVS docet רחנ dיר diftinguere, quia Exod. VII, 19. et VIII, I. locum inueniat. Nos approbamus hoc, et obferuamus: quod vox 7 ma $f 8$ abfolute 


\section{DE PAF YRO FRV TICE.}

et aisticos ponatur, Euphratem, רואי vero ei e regione pofita, Nilum fignificet, id quod cuilibet fedulo Prophetae noftri Lectori non erit incognitum. Illufrabimus hanc obferua: tionem Exegeticam exemplis.Sic Ef VII,20.c.VIII,7.c.XI, 15. Euphrates vocatur רa prater capitis noftriXIX,5.vbi res patet. conf.Gen. XXXI,21.Ex. XXIII, 3r, et in emphafi Gen.XV, 18.Contra רואי Nilum notare fenfu latiori.probat c.XXII, et nofter locus, add. Gen. XLI, I. Exod. I, 22, Ier. XLVI, 7.

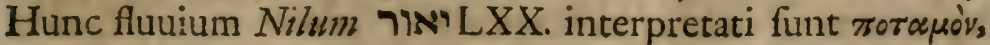
et recte, fiquidem alii Scriptores eodem nomine eum dignantur. AELIANVS Var. Hiftor.Lib. I.c. 4. de cane Ae-

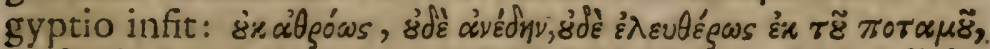

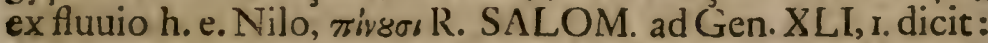
Aegyptum fuifie יאורים יאורים foffis frequentem, et aquas Nili in eas exundaffe, atque inde Nilum dici רiא:. Sunt, qui nomen 17 ?ר Iordanis ex compofitum, qua fí riuum Dan dicas, quod Geographi tradunt, eum iuxta vrbem Dan fuum ortum habere, quod tamen obiter obferuamus.

\section{XVI.}

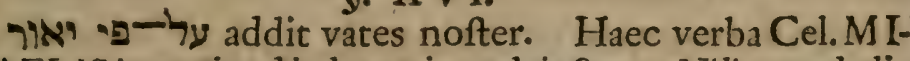
CHAELIS in notis ad h. l. vertit: ad ipfum os Nili, quod alibi dicitur labium, vti Gen. XLI, 3. vbi Pharao videbat feptem

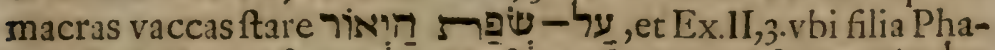
ranis, facta capfacannea, Mofen infantem pofuit $\overrightarrow{2}$ רiאi- ad labium feu os fluminis. De capite et ore Nili tanta dubitatio fuit apud veteres, vt locum dederit prouerbio, et LVCANVS dicat:

Arcanum natura caput non prodidit vlli:

Nec licuit populis paruum te, Nile, videre:

Amouitque finus, et gentes maluit ortzs Mirari, quam nofje tuos.

H. I. Stroomien.

6. XVII.

yרומ pheta? Lege HERODOTVML, II.p.137. qui poftquam de 
loto verba fecit, alios etiam memorat fluuiorum et paludum

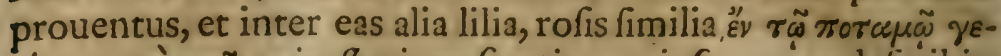

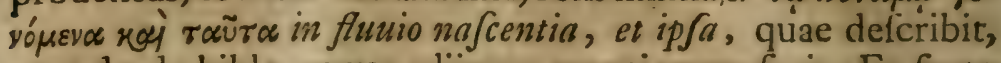
quando de biblo annua, aliisque mentionem facit. Et forte tamen rectius cogitamus hic de lino, Aegypti fingulari proventu, iudicat VITRINGA ad h.l. Nos vero latiore fenfu accipimus ע פ dẹ fatis cuiusuis generis; habet enim Aegy-

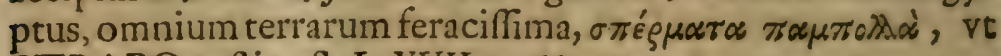
STRABO teftis eft L.XVII.p.sor.

\section{XVIII.}

שב generatim arefcendi notionem habet. Speciatim et quidem proprie vfurpatur tuni de exfecatione mav is Iof.II, 10. et fluuii Iob. XIV, 2. P. L, XXIV, ז5. et cap. noftro XIX, ร. tum de exficcatione vegetabilium, quae, abfumto fucco natiuo, omni decore priuantur, vt graminis Iob. VIII, I2. Ief. XL, 7,8. herbae agri ler. XII, 4. arboris Ioel. I, I2. Senfus itaque, vti vult VARENIVS ad h. l. absque Geographia haberi nequit. Nimirum foecundatio et vita ער ערומ 5 dependebant a Nili inundatione, quae incipit circa 17.Iunii, et 40. diebus crefcit, totidem recedit. Quantum igitur creuit Nilus, tantum fpei in annum eft Aegyptiis. Quidquid autem fluuius non adiit, ficcum ac fterile iacet. Et hoc 5 h.l.obuenturum effe praedicit Propheta. Siquidem Nilum exariturum effe v.5. 6. monuerat, quod tamen de diminutione, non omnimoda exficcatione effe intelligendum ex hiftoria probant THOM. AQVINAS in Comm. adh. 1. et IO.COCCEIVS T.II. Opp. Comment. in Ef. ad c. XIX, 5. 6. \$. I8. f. 285 .

\section{XIX.}

Denique vociferatur Propheta quafi vehementiore fpiritu ๆา $A \int y$ ndeton enim quilibet videt. Vix pofuerat $\mathcal{Y}$ ? adicit ริจ Haec vox autem notat propellere, propulfare. Proprie difpulit inftor fumi euanefcentis : dicitur enim de vento, qui propellit fumum et alias res leues, quas facile poteft tollere et abripere in altum, vt funt paleae, quifquiliac, ftipula et in h. 1. femen. In eadem forma et fignificatione hac voce vtuntur reliqui fcriptores facri, Iob. c. XIII, 25. Prou. XXI, 6. PS.I, 4. Nos cum aliis peculiarem emphofin obfcrua- 
mus in voce ๆ.2, quam vidit quoque interpres Graecus,

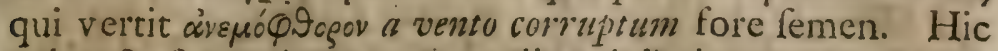

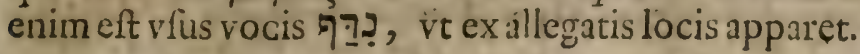

\section{§. XX.}

Ex his explicatis verbis emergit hicce fenfus proprius Propherae noftri, quo praedicit, magnam in Aegypto fufcitatum iri tempeftatem, quac Nilum repelleret, riuos et dí́su$x$ as cius propemodum exficcaret, et fiutecta omnia, qualia ענ ערות inprimis erant, et omnes infuper proventus Nili ita frangeret, difpelleret, disiiceret, vt plane perirent atque abolerentur. Senfum myfticum et figuratum in verbis noftris non quaerimus, quippe inftituti noftri memores, qui de ערות commentari coepimus. Qui vero hac de re pluribus vult edoceri, adeat VITRINGAMud cap. hoc XIX. Ef.f. 70 . fqq. et f. 747 . fqq. in Comment.

\section{XXI.}

Emenfo labore Critico, fatisque ex fontibus Ebraeorum enodato nomine ערות, quod Latini papyrum vertunt, vti euicimus; neceffe eft, vt labores phyficos fufcipiamus, et de nat un "I ac indule fruticis huius quaedam exponamus, ad quam quidem operam nos vocat

\section{CA PVT II. \\ DE CONSIDERATIONE PHYSICA ת ת SEV PAPYRI. \\ $\Sigma \Upsilon N O \Psi$ I $\Sigma$.

Quadam circa boc Caput monen- Secundo folia $₫$ VII. tur. $\$ I$. Ante ommia audicndus locus ex Plinio S II.

$V$ nde eruitur definitio papyris III. Additu diutifo in partes. integrantes $5 \mathrm{IV}$. iuxta unatomiam plantarum a recentioribus praefcriptam $\oint V$.

Primo loco explicatur cacumen G.VI. Tertio caude $x$ fou caulis $\S$ VIII. Eiusque fubftantia interna t. quoad fiftulas fucciferiss $\S I X$. t. quoad tracheas f.poros aërcos $\delta X$.

Et externa, vbi exponitur Figura papyri $₫ X I$. Longizudo papyri $\oint$ XII. Cortex papyri $\$$ XIII.

Quarto 


\title{
DE PAPYRO FRVTICE.
}

Quarto medulla f. Juccus papyri Septimo nutrimentum buius fror 6 XIV.

Quinto fibrae ligneae caudicis pa- Denique fc men, quo deftitui papyfyracei $\oint X V$.

Sexto radix pafyri $\$ X V I . \quad$ Inde tranfitur ad Caput III. $\oint$. XIX.

\author{
S. I.
}

Dim ad delineandam papyri naturam pedem mouemus, duo praemonemus Beneuolum Lectorem, primo, nos limites a Iefaia nobis pofitos, haud quaquam effe migraturos; Quum itaque ille de Aegypto agat, nos quoque non nifi de papy yo Aegypti celeberrima verba effe facturos, quantumuis alibi quoque papyrus extiterit; deinde nos omnem de hac materia certitudinem probabilitate hiftorica effe confirmaturos, quippe cum aliter rem hancce intricatam explicare nequeamus.

\section{S. II.}

Agedum L. B. audias, quaefo, omnium primo,quomodo PLINIVS in Hift.Nat.L.XIII. c.XI. T. III. Sect. XXII. p. 79. Edit. FRANC.MVGVET, Parif I685 nobis defcribat papyrum.Papyrum, fic habent eius verba, mafcitur in paluftribus Aegypti, aut quiefcentibus Nili aquis, vbi euagatae ftagnant, duo cubita noin excedente altitudine gurgitum, brachiale radicis obliquae craflitudine, triangulis lateribus, decem non amplius cubitorum longitudine in gracilitatem faftigatum, thyrfs modo cacumen inchudens jemine mullo, aut vfus eius alio, quam for is ad Devs coronandos. Radicibus incolae pro ligno vtuntur: nec ignis tantum gratia, fed ad alia quoque vtenfilia vaforum. Ex ipfo quidem papyro nutigia texunt: et e libro vela, tegetesque, nic non et veftem, etian ftragulam, wi funes. Mandunt quogue crudlum, decoctumque, fuccum tantum deworans. tes. Hactenus Plinius.

\section{s. 111.}

Papyrus itaque Aegyptia eft frutex ille Nili, qui cau: dice triquetro, quatuor cubitorum alto, et in multas membranulas fecabili, foliis gladiolo quoad acumen fimilibus, finc femine, et fine fructibus, ex littore procrefcit, infra terram autem radices decem cubita longas agit, ad varios vfus victus et amictus Aegypriorun.

S.IV. 


\section{§. IV.}

Diuifionem papyri, quod attinet, SALMASIVS, vir magni nominis, deque PLINIO emendando immortaliter meritus, papyrum diuidit in communem f. vulgarem, et excellentem $f$. Niloticam, prouti fupra ad Cap. I. \$. XIII. iain obferuauimus. GVILANDINVS pariter papyrum dupliciter diuidit. Papyrus, inquit, eft duplex, vna, quae proprie vocatur biblus Aegyptia, non nifi in Aegypto et India Straboni nafcens: altera, quae extra Aegyptum in Italia etiarn gignitur,et a THEOPHRASTO Sari,ab EVSTATHIO biblus altera appellutur, de quo fupra ibidem dictum eft. Nos quidem in praefens huic diuifionis explicandae labori fuperfedebimus; fatis enim nobis videtur partes omnes, ex quibus papyrus noftra, quam vates per תing intellexit, componitur, in quasue diuiditur, adduxiffe.

\section{S. . V.}

Quumque papyrus noftra merito ad plantas referatur, volupe eft anatomiam quafi quandam huius fruticis, fiue, qui eum ita nuncupare malit, huius plantae inftituere. Ad hanc vero methodum egregia cum laude glaciem nobis fregere recentiores Philofophi. Primi quidem anatomiam plantarum tradiderunt MARCELLVS MALPIGHIVS, Italus, a) et NEHEMIAS GREW, Anglus. b)Eandem vlterius excoluit ANTONIVS van LEEWENHOECK ac Celeberr.WOLFFIVS in Philofophia fua Experimentali, quam eamdem per anatomiam folioram perfeciffe legitur THVMMIGIVS. c) Praeclarum hunc ordinem, praeclaram hanc plantarum in partes integrantes diuifionem nos quoque fequemur.

a) in anatome plantarum, quae idea hiforiae philof plicas de Partibus II. conftat, I. prodiit plantis et variii lectionibus aliis Londini 1675. II.vero 1680. ibid. edita Londin. 1682. ful.

b) in anatomia plantarum, cum c) in Act. Erudit. A. 1722. p. 24. \$. VI.

Primum vero hic merito fibi vendicat locum cacumen papyri. Frutex videlicethicce fififam panniculam in funmitate habet, $h . e$, denfa et opaca coma gaudet,et cacumen 
gracile ac acutum includit, denique vero in conum definit, qualis in acantho, a SALMASIO p.380. 1. c. adpicto,adparet. Hanc papyri fummitatem STRABO $x$ csi $\tau \eta v$ adpellat, quando papyrum $\chi \alpha^{i} \tau \eta v \varepsilon^{3} \pi^{\prime} \alpha^{\prime}$ re $\rho^{8}$ habere tradit; PLINIVS vero figuram thyrfi, quandoquidem papyrum, ait, eft in grucilitatem faftigatum, thyr $/ 2$ a) modo cacumen includens l.c.

a) Thyrforum nou eft vina figura in antiquis monumentis. Aliis x) veluti globus impofitus confpicitur. Hinc, quod THEOPHRAS'TVS in Colocafia fiue Faba Aegyptia rodóa megipeè̀s i. e. caput rotundum, globofumque, vocauerat, quodque

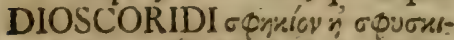
ov dictum, PLINIYS L. XXI, c. XV. aperte thyrfum nuncupat, quod bene obfernat GVILAND. 1. c. p. 218 . n. 23. Ex quo cernere elt, quod PLINIVS perthyrgmum caput, rotundam ac denfam comam intelligat. In THEOPHRAS'TO autem, ex quo PLINiVS omnia haufit, riec vola nee vefigium de eo occurrit. Alii thytfos 2) pro caulibus plantarum acceptarmat. SERVIVS ad XII.

\section{VII.}

Folia papyri iuxta VESLINGIVM ad Profp. Alpin. de plantis Acgypt.c. 36. circa vtrumque fapi extremuni, inferius ampliora (purganii a). cuperiue inftar funt repanda : fuperne minora, quem radiatum veluti calicem innafcenti iubae b) fubiiciunt. GVILANDINVS vero p. 222. vult folia typhae c) paluftri effe fimilia, fiue vt dicit Elkaui (funt verba GVILANDINI) in libro de fimplicibus medicamentis, duobus locis c. r47. et c. 547.gludiolo, minime tamen acuta, fed ubtufa. De'his foliis EDVARDVS LEIGH inCrit. S. N. T.p. 60 ad rocem Bißnos commemorat, veteres non- 
nunquam in is fcripfife, de quo altum apud alios filen. cum.

a) Sparganium medicis eft notiffima herba, vulgoqueSchwertel-Kraut a figura cognominatur. Cyperius vero eft fpecies iunci odorati. Hoc nomen VARRO R. R. III, r6. COLV. MELLA XII; 20 . aliique in genere neutro vfurpartunt. PETRONIVS c. 127. canit.

Enicuere rofae violaeque et molle cyperon.

Vetuftifini Graeci xüreigov femper dicunt, THEOPHRAS'IVS, THEOCRITVS aliique. Graeci pofteriores หu่ $\pi$ gov maluerunt feribere, inter quos DIOSCORIDES et PLI-

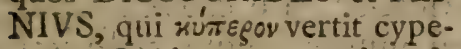
rum. Quid vero proprie per cyperum intelligatur, variae proftant fententiae. Quidam ex antiquis Grainmaticis i) filer. effe interpretati funt; alii 2) gladiolum, ab acumine $\mathrm{fci-}$ licet foliorim dictum. Nobis perplacetSALMASII iudicium, quod eft: aut vno eodemque nomine rvтsige duae diuerfae plantae vocatae funt, quod non mirum eft; : ant certe Latini

\section{§. VIII.}

Cacumen excipit caulis f. caudex papyri, qui ex radicibus afturgit, et folia cum ramis fuftentat, fuccumque nutritium ex radice fingulis aduehit. Hunc defcribamus, oportet, tam quoad fubftantiam internam,quam externam. \$. IX.

Subfantia eius interna multis procul dubio gaudet fiftulis fucciferis, et tracheis feu poris aethereis. Fiftulae fucciferae a medullac centro verfus peripheriam caudicis $\mathrm{C}_{3}$

vsque 
vsque ad corticem excurrunt, quae inftar venarum transuerím fectae fuccum effundunt, iuxta morem ceterarum plantarum, quod THVMMIGIVS erudite obferuat in Inftitut. Philofophiae Wolffianae T. I. Part. IV. de plantis f. vegetabilibus c. r. \$. 275. et 277. idque ex GREW Anatomia plantarum, qui Figuras addidit Tab. LI. LII.

\section{๖. $\mathrm{X}$.}

Tracheae f.pori aërei concauant papyrum, et fpongiofam ac fungofam eius reddunt fubftantiam. Quae omnia GVILANDINVS l, c. p. 206. vbi fcapum ac firpem, h.c. caudicem papyri defcribit, confirmat. Digna funt eius verba, quae hic legantur. Eft $\int c a p u s$, inquit, ex rara et fungufa fubfania conftitutus, cuius biantes et laxae interius caututes copiofo bumore explentur, qui fimul atque euanuit, tota caulis craffities concidens, redditur mulio, quam erat, tenuior ac Jubtilior. Et rurfum alio loco p. 360 . vbi explicat, quid per fungum papyri debeat intelligi, nempe fcapus, h. e.caudex papyri, a quo philurae abripiuntur, qui multis vbique inanitatibus inftar fungorum hiet.

\section{§. XI.}

Subftantiam externam caudicis papyracei PLINIVS no1ter l. c. defcribit a figura et longitudine. Figura externa dicitur triangularis, quod etiam affirmat VES LINGIVS l.c. Hinc enucleator papyri magna exemplorum farragine refutat ARISTOTELEM, qui Lib. XVI. Problem. 8. negat, vllius plantae caules truncosue forma triangulari aut multiangulari confare. Idem VESLINGIVS papyrum habere caulem numerofum 1.c. ait, vnde caufa fortafis adparet, cur vates nofter in numero plurium climet:

\section{S. XII.}

Longitudo huius caudicis triangularis a PLINIO decem $\mathrm{cL}$ bitorum efle dicitur. $A t$ vero SALMASIVS ad SOLINI Po1yhift. p.379.eum hallucinatum cffe laudabiliter animaduertit. Nullo eft, inquit, in verbis Plinii tran $\int p$ ofitiv, vt putat Guilandinus, fed ballucinatio Plinii, qui radicis longitsdinem papyro ipfi, i.e. cauli tribuit. Atqui Theophraftus radicem 10. cubita

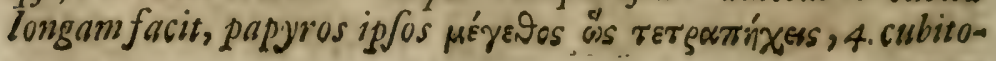


rum longitudine. Sed ille baec mifcuit et confudit iure fuo ac more perpetuo. Quod ctiam verofimile videtur, cum cacumen pro fuo lubitu explicauerit PLINIVS, et cum fruticem ıo. cubitorum proceritatem attingere vix fit probabile, alioquin enim non frutex, fed arbor effet. Notandum tamen, quod VESLINGIVS fpeciofam proceritatem 7 .cubitorum 'papyro tribuerit.

\section{S. XIII.}

Sed nec practereundus eft ipfe.Cortex papyri, quém nunc cutem, nunc corium vocat Plinius. Hic fuit lenis, glaber, et admodum tractabilis ac flexibilis, id quod exinde colligitur, quonia in nauigia, vela, veftes ac funes, et quae funt reliquia, ex eo contexta referuntur, de quo pluribus in Cap. feq. noftrae Diflertat. diflerendi locus erit.

\section{XIV.}

Medulla a) f. fuccus caulis Nili accolis ad manducationem inferuiebat. Succus ille, quem mandunt, iuxta VRSINVM in Arbor. Bibl. p. 159. aut eft, quod dicunt, faccharum, aut fimile, vt et papyrus annumeratur arundinibus faccharinis, quibus in Aegypto nihil effe frequentius aitBELLONIVS L.II. Obferuatt. c. 34. Quin et Indicae cannae, quibus delicatiores pro fcipionibus vti folent, et folia illa, quibus faccharei, ve vocantur, panes, Zuckerhüte, inuoluuntur, delati ex infulis Diui Thomae et aliis, de papyri genere effe dicuntur, vt notat MAT T HIO LVS in Divlcorid. L.I. c. 98. De faccharo veterum et noftro vid. THALMVTH.in Nou. Repert Tit.V.

a) quae in aliis plantis ef con- Microfcopicas in Philofophia geries veficularum minorum, Experimentali. conf. THVMnitis albedinem aemulantium, MIGIVS p. 340 . in Inftit.Phil. iuxta WOLFFII Obferuatt.

\section{§. XV.}

Fibrine lignese caudicis papyri, funt fafcicīli plurium minorum ftupendae prorfus exilitatis. Hinc etiam in multa fruftula, in multas membranulas fecabilem defcripfimus pa. pyrum, quod confirmat PLIINIVS de confectura chartarum. Praeparantur, inquit, ex papyro clartae, diuifo fcapo in proetenues, fed quam latifinas ploiluras. Principutus medio, atque inte 
inde fiiffurcie ordine. Nunquam autem plures fapo pbilurne, quam vicenae. l.c. Quod it itaque caudex vnius fruticis papyracei in viginti membranulas feu philuras diuidi potuit, quod acu feciffe teftantur quidam Codd. Plinii; exinde procliue dictu eft, fibras ligneas papyri tenuitatis mirificae fuiffe. Aiu autem has fibras et haec ramenta effe diffecta ; vix videtur probubile, quantumuis MONTFAVCON in Antiquitat. Rom. T.III. P. II. L. V. c. 5. P.352, eamdem amplectatur fententiam. Vero magis congruum eft GVILANDINI

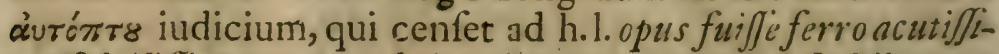
mo fubtilifimoque, tres cigitos lito, quo ramenta f philurae a fcapo diuelli potuerint, acu eam ad rem nibil emolumenti praefunce, fed inutili prorfus abiiciendaque.

\section{S. XVI:}

Radicem papyri quod attinet, ea eft multiplex, vt in reliquis fruticibus. Merito autem diuiditur in realam et obliquam ab eruditifimo SALMASIO. Radix recta dicitur aequare craffitudinem bracchii humani, et longitudinem 10. cubitorum, vti fupra monitum hoc Cap. \$.XI. Obliquae vero, quae ex recta radice prodeunt, atque in limum emittuntur, funt partim tenues, partim denfae. Et fic recte emendauit PLINIVM in radice explicanda obfcurum. Namque ille 1.c. papyrum brachiali radicis obliquae craffitudine ex Nilo afcendere narrat, cum tamen THEOPHRASTVS, ex quo haec haufit rerum naturalium infelix conditor, dicat: $\pi \alpha-$

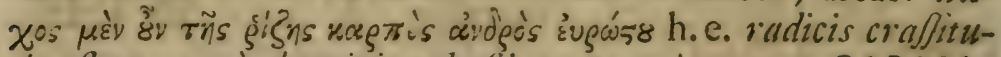
do eft rt ragrtics bominis robufti. Kagriós autem, SALMASIO iudice, notat non tam brachium, puta pulpan iuxta cubitum, quam potius primam partem palmae, ut CELSVS loquitur.

\section{§. XVII.}

Nutrimentum ac vegetatio papyri fruticis inprimis repetenda eft ex aqua Nili fluminis, id quod iudicatu inde facillimum $\in \mathbb{f}$, quoniam papyrus, aefu folis ficca, et ad texendum inhabilis facta, admota aqua tractabilior fuit reddita. Praeter aquam autem ad eiusdem nutrimentum terram ciusque falem ac fulphur non paucum attuliffe, inde con- 
Atat, quia ex littore procrefcit, et eius radices obliquae in fimum terrae emittuntur. Succum vero nutritium non modo a radice per caulem in folia, fed et a folisis per eundem caulem deorfum in radicem moueri laudabiliter obferuauit MAIOR, Prof. Kilonienfis in DifJ. de planta monftrofa Gottorpien/s, et pluribus experimentis confirmarunt PERRALTIVS in tentamine de circulatione Jucci nutritii, nec non MARIOTTVS in tentamine de vegetatione plantarum.

\section{XVlii.}

Denique quod Semen plantarum attinet, noftrae aetatis Philofophi, tantum non omnes, cunctis plantarum generibus femen attribuunt, quod recentiffimo MALPIGHIVS experimento oftendit, vid. WALCHIVS in Lex. Philofoph. p. m.1962. Atvero de papyro noftra expreffis vcrbis PLINIVS eam nafci fane femine affeuerat, quod et eius Commentatores non inficias eunt. Hinc etiam flores ac fructus ex papyro fruftra quis exfpectabic.

$$
\text { §. XIX. }
$$

Et haec fufficiant de natura ac partibus papyri, vbi verba noftra nemini obtrudimus, fed cuiuis limatioris iudicii ingenio ad ulteriorem difcuffionem relinquimus; nos in hac quoque parte alterius inftitutionem vltro flagitamus. Sed pergendum nobis eft ad

\section{CAPV'T III. \\ DE CONSIDERATIONE HISTORICA$$
\text { ערוֹ SEV PAPYRI. }
$$$$
\Sigma \Upsilon \mathrm{N} O \Psi \text { I } \Sigma \text {. }
$$

Multi biforiam plantarum fcripferunt $\$$. I.

Hoc Caput III. etiam eft mere Hifloricum \$. II. et tria exponit de papyro, nempe biforiam

I. Natiuitatis f. ortus, qui $\mathrm{cfl}$ ad Nilum pajyrifertum S. III. quod probatur ex Hifloringraphis $\oint$. IV. nons autem dcmum

tempore Alexandri M. qui Aeg.yptum cepit, et Alexandriam condidit, papyrus fuit cognita, Sed ante, S. $V$.

II. Vtilitatis, qui campuseft ampliffimus $\boldsymbol{S}$. VI. vitilis autem fuit papyrus

1) ad indumenta corporis $\int$. vefles D. VII. 
2) ad chartas faciendas, vbi exponitur modus chartas ex papyro faciendi . VIII.

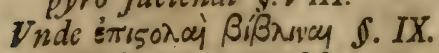
Et diplomata ac libri papyrei bodienum fuperfites $\oint$. X.

Erant etiam notae boni papyri, ex papyro frutice confecti $\hat{\hat{j}}$. Xi.

Exteri quid cum papyro Aegyptiorum fec rint $S$. XII.

Et inde noftram chartam Jcriptoriam inuentam efje baudinepte a multis colligitur $§$. XIII. officina illa papyracea Aegypti, quando deficrit, docețr H.XIV. 3) ad texenduns

* copbinos $\S . X V$.

I nauigia $\oint$. XVI. illuftratur boc arculaMofis, quae vtrum Chriftum praefigurauserit? quaeritur \&. XVII.

a) funtes, vela tegetesque $\$$. XVIII. 4) ad edendum, non aeque ad bi- bendum §. XIX. Vnde quinam fint papyropbagi? S. XX. non ad bibendum ; vbi quaeritur : quid fit vinum biblinum? S. XXI.

s) ad armaturam nauticam f. XXII.

6) ad calceos facrificulorum $\int$. Ala. minum \$. XXIII.

7) ad coronandos Deos \& XXIV.

8) adfrata quoque varii generis 6. $X X V$. qualia funt
a) ceruicalia $\oint$.XXVI.
B) tori mortuorum farciendi \$. $X X V I I$.

9) adignem.

\section{t. in candelis $\oint$. XXVIII. \\ t. in calefaciendo $\$$. XXIX.}

Nec bocmirum effe, illuflratur igne bonuris, Carolo V. accenfoib.

IIl. Operariorumaliorumque quaeftum ex papyro quaerentium $\S$. $X X X$. Inde tranjiturad Cap. IV. §. XXXI.

\section{I.}

Vlti haud perfunctoriam in confcribenda de plantis 1. Liftoria operam induftriamue collocarunt, fue refpiciamus veteres, frue recentiores. Ex veteribus procul dubio THEOPHRASTVS eminet ac PLINIVS, ille quidem in hiftoria de plantis, hic in hiftoria naturali. Ad recentiores autem merito rcferuntur I) VLYSSES ALDROVANDVS in Opere, quod de dendrologia concinnauit, quodque ob raram eruditionem aliquot vicibus prelo fuit recufum. 2) Clarifl:DEDV, qui de l'ame des plantes,effais de Phys 2que Parif 1682. 12. edidit, vbi doctiffimus auctor fententiam de generatione plantarum per ouula feminis, quae in ceteris animalibus deprehenduntur, acerrime propugnanit. 3) Addendus ef IO. RAIVS in hiftoria plantarum, cuius Pars I. Londini 686.fol. Pars 11. vero 1688. lucem adfpe- 
sit, et 4) GEORGIVS A TVRRE in hiftoria plantarum, Batau. 1585 . fol. vt aliorum recenfionem h. l. omittamus. §. II.

Hoc tertium Caput noftrum pari ratione mere eft Hiftoricum, omnia enim, quae de תing h.e. papyro memora. tu.digniora funt vifa,complectitur.Maximam autem eius partem enarratio vtilitatis abfoluer. Vt vero eo melius Lectori de ordine noftro conftet; trademus initio hiftoriam natiuitatis; deinde hiftoriam vilitatis ; denique etiam hiftoriam operariorum, aliorumque, quaeftum ex papyro et victum quaerentium adponemus.

\section{§. III.}

Hiftoria NATIVITATIS originem et ortum papyri nobis commemorat. Vbi autem papyrus nafcatur ex capitibus antecedentibus iam poteft intelligi. Obferuauimus enim Cap. 1. §. X. quod papyrus in Syria, nec non circa Euphratem, rurfus in Chaldaea, tefte GVILANDINO, olim fuerit inuenta; optima vero ac praeftantilfima papyrus prope Nilum progerminauit, quod vates nofter liquido nos edocuit fupra, cum exclamaret ערות על יאיר ibid. §.XV.

$$
\text { §. IV. }
$$

In eadem veritate veteres Hiftorici nos confirmant. PLINIVS enim fupra l. c. expreffis verbis dixit: papyrum nafcitur in paluftribus A egypti,id quod THEOPHRASTVS longe ante eum prodidit. Et VESLINGIVS ad Profp. Alpin. c. 36. fcribit: papyrum affurgere ab arundineis fibrofisque radicibus circa Nilififagnantis ripas. Quid? OVIDIVS, Ni-

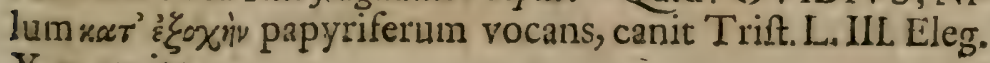
X.v. 27.ita:

Ipfe papyrifero qui non anguftior amne, Mifcetur vafto multa per ora freto. S. V.

Nemo autem fibi perfuadebit, papyrum fruticem demum tempore Alex. M. quo A geg ptum occupauit,et Alexandriam vrbem condidit, annos circiter 330. ante C. N. in $\Lambda$ egypto fuife repertum, vti quidem M. VARRO credidit, quod idem fatetur AVGVSTINVS de C. D. L. VI. a cuius fententia 
quioquie EVSTATHIVSadXXI.Odyffeae Rhapfodiam non abhorruit; quin imo eandem opinionem LAVRENBERGIVS in Acerrae fuae Centur. III. Hift. so. tuctur. Sed falfo. Papyri enim vox vetuftiflimis Graecorum fcriptoribus fuit notiffima.Effe ex antiquiffimis ANACREONTEM, lyricorum Carminum fcriptorem longe fuauifimum, fatis liquet teftimonio HERODOTI et PLA'TONIS, qui in Phaedro eum ooфs etiam nomine dignatus eft; hic de femet ipfo loquens, haec protulit verba:

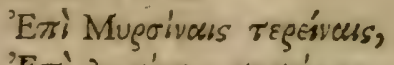

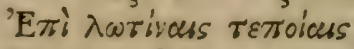

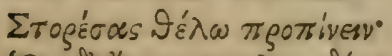

"O $\delta^{0}$ "

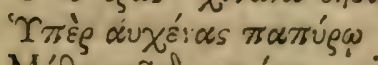

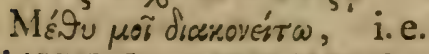

Teneris fuperque myrtis, Super et virente loto Recubans bibam libenter: Religatus at pap.yro Tunicam, cupido collo Mibi poculum miniftret, vti HENRIC. STEPHANVS vertit. Nauium papyracearum, et arculae Mofis, ex papyro fine dubio longifime ante Alexandrum factae, nullam iniiciemus mentionem. Plura vide fis apud GVILANDIN. Membr. II. p. 32. et fqq.

\section{S. Vi.}

Venio nunc ad vberrimum campum vTILITATIS papyri. Obfupefcat fane, quisquis audiat, aut legat commoda, quae ex pupyro frutice ad Áegyptios redundauerunt. Nos breuifimis, quantum eius fieri poterit, vtilitates varias papyri recenfebimus, quippe cum nos in eo THEOPHRASTVS apud BOCHARTVM Hieroz.P. I. L. II. c. XXXIV. f. 342. confirmet.

\section{§. VII.} bat partibuis,

Vtilis autem fuit papyrus pro varis, ex quibus confa-

I. AD VESTIENDVM ORNANDVMVE CORPVS. HinC BVXTORFF. in Lex. Chald. Thalmud. et Rabbin. p. 1780. ad vocem רפפ ex cortice, inquit, in tenuiffimas plagas folutu, fiebant tegumenta, quae imponebuntur mortuis in Beref. Rabb. Sect. XXXVII. Sic in Talmud. Hierofol Maffech. Sota cap. vltimo in fine: raceas fc. veftes. Gloffam addic BVXTORFF. 1. c. hancce: [pecies herbae eft (papyrus,) quae cortices habet, ct faciunt ex ea veftimentun ad ornatum.

\$.VIII. 


\section{§. VIII.}

II. AD CHARTAS FACIENDAS, ET INDE AD SCRIBENDVM. Egregie modum nobis exponit faepe laudatus GVILANDINVS I.c. p. 32I. Philurae f. pagellae erant a caule papyri difractac, ex quibus, alteris recto fitu fubftratis, et alteris transverfa pofitione fuperiniectis, chartae conficiebantur, vel, vt PLINIVS ait, texebantur, praebente glutinis vicem Nili aqua, non illa clara et limpida, fed turbido liquore, ac pingui terrarum fucco coinquinata. Et p. 324. pergit : porro autem textae fiuc compofitae ad eum, quem dixi, modum, pagellae ftatim fupponebantur prelis, et valide comprimebantur, $v t$ redundans aquae humiditas emanaret atque defluerct, quod auctor, PLINIVS, ficcare appellat, demumque in vmbra arefiebant. Ad extremum malleo complanabantur chartae, quae que diuturniores effe debebant, bis glutine attrectabantur, et malleo bis tundebantur. Afperitas vero papyri laeuigabatur dente conchaue, et fic litterae calamo fuperfcriptae combibebantur.

\section{§. IX.}

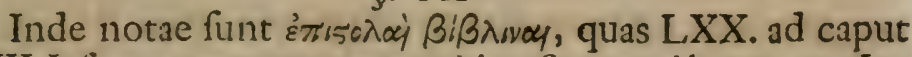
XVIII.Iel.v. I. commernorant, vbi vafa papyri leguntur. In-

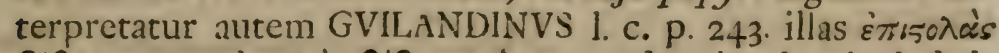

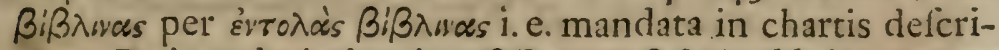
pta. Pari modo inde etiam $\beta / \beta \lambda \alpha$, et $\beta, \beta \lambda i \alpha$, libriex papyro feu biblo conuoluti, notifima funt Graecorum nomina. Conf. THEOPHRAS'TVS de Hift. plant. L.IV. c. 19.

$$
\text { 5. } \mathrm{X} \text {. }
$$

Proftant etiamnum hodie diplomata, proftanc et libri ex papyro facti, fed rari, quales Celeberr. MONTFAVCON vltro citroque vidit variis in locis et obferuauit. Sic refert, in Chartulario S.Dionyfii in Francia adeffe diploma graecum papyreum, quod nobis in Palueugrapioia Graeca p. 15. publicauit. Ibidem pergit: in hoc chartae genere fcriptum eft Euangelium S. Marci Venetiis, fed valdopere putrida funt eius folia; Iosephus in Bibliotheca Ambrofiana Mediolanenfi, papyreis toliis defcriptus, longe meliori eft conditione. Sed fingulariffimus, et ipfa foliorum integritate prae- 
ftantifimus omnium liber papyreus Epiffolae S. Augufini offe dicuntur. Nam ii, qui hunc librum fcriplere, cum materiae fragilitatem compertam haberent, poft quaterna vel quina papyrea folia vel vnum vel duo membranacea folia interferuere, in quibus fcripturam continuarunt, quo factum eft, vt papyrea folia integra manferint, fic loquitur vir incomparabilis in Antiquit. Rom. T. III. P.II. L. V. c. $5 \cdot$ p. 35 . fq. Binos libros Latine fcriptos papyreos memorat quoque MABILLONIVS de re diplomatica L.I. c.8.

\section{XI.}

Habebsnt etiam Aegyptii certas notas bonarum chartarum ex papyro confectarum, nempe, fi vel fcabriores glabriorefue effent, vel haufto liberalius Nili humore, negligenter ficcatae eflent, malleo, odore, et oculis illud deprehendebatur, quandoquidem, fi quis folium chartae ad folis lumen obuertiflet, non difficile erat, latitantes inter philurarum compactiones, feu coniunctiones, maculas confpicere, ceteroqui cognitu obfcuriores, tum ob fungofam, bibulamque papyri bracteam, quae humorem laxitatibus fuis occultabat, tum quia praelentiam fuam non fatebantur, nifi ob lituram, delendi expungendique propofito inductam, vti GVILAND. autor eft p. 3i9.

Quae chartae a confertoribus in Aegypto negligenter exficcatae fuerant, cae apud gentes exteras nouo labore retexebantur, quae, quia deftituebantur Nili aqua, accipiebant pro glutine, tenui]/zmum farinae tritici pollinem, feruente aqua, minima aceti afperfione perfufum temperatumque. Atuero fic ad fragilitatem vfque durefcebant, nec tuto ad conuoluenda et complicanda volumina adhibebantur, tefte DALECHAMPIO in Plin. ad h. 1.

\section{§. XIII.}

Et inde natales fuos noftras chartas, ex variis linteis, et laceratis panniculis productas, traxiffe, haud inepte cum ex nomine cius, tum ex paritate modi, eam conficiendi, colligunt rerum inuentarum conditores. DALECHAMPIVS 1. c. et SALMVTH. PANCIROLL. in Nouis Repert. Tit. XIII. 
XIII. hodie, aiunt, ob ftrenuam incolarum inertiam aliunde aduectis chartis, ars haec Aegypto natales tantum fuos, non aeftimationem debet, add. VRSINVM in Arbor. bibl. p. 158. qui et VESLINGIVM et GVILANDINVM his merito adiungic. Diuinum fane inuentum eft papyrus, $f$. charta noftra fcriptoria, et videtur inuentor ab Aegyptiis inuentionem accepiffe. MONTFAVCON autem noftram chartam ad bombycinam in fimilitudine ex detricis pannis confici coepiffe affirmat in Antiquit. Romı.l. c.p.353. et in Palaeographia Graeca L. I. f. I3. fq. Sed cum adhuc fub iudice lis fit, noftrum fane haud eft tantas componere lites, praefertim cum de תוาบ f fribamus, non de charta noftra moderna.

\section{XIV.}

Officina illa papyracea Aegypti defiit cum Sec. VII.quo Aegyptus in poteftatem Arabum deuenit, vti fcribit paulo ante citatus MONTFAVCON in Palaeograph. p. 19. quod probat ex EVSTA THIO, qui ad HOMERI Odyff. $\emptyset^{\prime}$.iam eo tempore chartae Aegyptiacae conficiendae artem defiiffe memorat, clare enim de $A$ egy ptiis fui temporis EVSTATHIVS

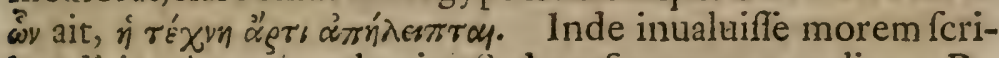
bendi in charta bombycina.Sed perfequamur et reliqua. $\mathrm{Pa}$ pyrus frutex

\section{S. XV.}

III. AD TEXENDVM etiam adhibebatur. Texebantur autem hinc $\aleph$. primo cophini $\int$. corbes, ad vuas e vineis colligendas apprime viiles. Huius rei fidem ex Hebraeis confirmat BVXTORFF. in Lex. 1. c. p.1781. Fiebant, inquit, et inde (ex papyro videlicet,) vafa texta inftar corbium, quorum vous fuit in vineis, Kelim. c. XVII.

\section{\$. XVI.}

7. deinde nauigia, non quidem oneraria, fed leuia, ac fi ita loqui liceat, volatilia." Horum meminit PLVTAR-

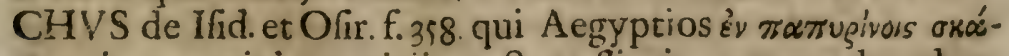
Qeø, in papyrinis nauigiis vectos efle ait, quemadmodum

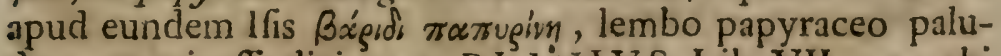
des pernauigaffe dicitur, et PLIN IVS Lib. VII. c. 22. vbi eadem appellat naues papyraceas, quas etiam $S T R A B O$ 
L.XVII. habet. Clarius autem C. PLINIVS alibi ait: in Britannico Oceano vitiles (fc. naues) corio (i. e. cute papyri) circumfutae funt in Nilo, ex papyro, et Scirpo, et arundine. L. VII. c. 56. Vitilem ex vimine nauem nobis depingit LVCANVS L.IV. de Bell. Ciü.

Canna falix madefacto vimine partum Texitur in puppim, caefoque induta ituenco, Vectoris patiens, tumidum fuperenatat amnem. Sic Venetus fagnante Pado, fufoque Britannus Nauigat Oceano : cum tenet omnia Nilus, Conferitur bibula Mempbitis cymba papyro.

Plura de his GYRALDV'S de nauigiis c.VII.afiert. conf.BELLON. L. II. Obferuat.c. XXX.

\section{§. X XII.}

Illuftrauit practerea naues papyraceas, ipfo fatente GVILANDINO l. c. p. 243. Mofis adhuc infantis in aquam

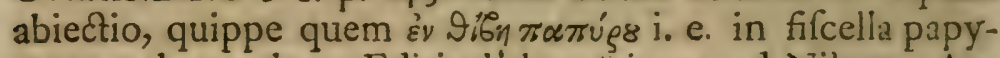
racea, vel, ve vulgata Editio habet, lcirpea, ad Nilum, Aegypti fluuium, expofitum fuiffe, legitur Exod. II. IOSEPHVS L. II. Archaeologias c. 5 . defcribens arculam, in qua Mofes in Nilum depofitus fuerat, rocat eam $\pi \lambda \lambda^{\prime} \gamma \mu \alpha \beta_{i} \beta \lambda$ wov $\varepsilon \mu-$

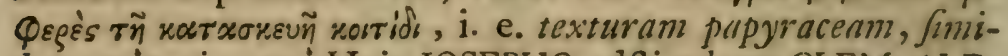
lem conipagi cunae. Huic IOSEPHO adftipulatur CLEM. ALEXANDRIN. L. I. Stromatum, in haec verba fcribens: $\varepsilon^{\prime} *$ Bi-

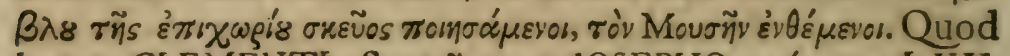
itaque CLEMENTI eft $\sigma \kappa \varepsilon \tilde{o s}$, et IOSEPHO $\pi \lambda \varepsilon \dot{\gamma} \mu \varepsilon$, LXX. fimpliciter $\theta \varepsilon \dot{\beta} \eta v$ appellauerunt. Num vero haec urcula Mo 2.5 Chriftum, in praefepio cubantem, pruefigurausrit? alia eft quaeftio ; meo quidem iudicio typi in Chrifum, praefertim tacente ipfa Scriptura, coaceruandi non funt, ne tandem, nefcio quis fenfus, facris oraculis affingatur. Porro

\section{§. XVIII.}

3. Tertio ex papyro etiam texebantur funes, vela, tegetesque, fed non nifi ex humido Jibro feu-cortice papyri. SALMASIVS, cuius nomen in orbe litterato magnum eft, l.c. p. 706. papyrum cum $\varphi \lambda \varepsilon \omega^{\prime}$, feu feirpo comparans, hanc veritatem immifcet: ex cortice interiore, fuse libro, funes, velo 


\section{DE PAPYRO FRVTICE.}

et cetera texebuntur. Recte itaque PLINIVS I. c. infit : poft hanc papyrum eft extremumque eius fcirpo fimile, ac $n \epsilon$ funibus quidem, nifi in humore vtile.

\section{§. XIX.}

IV. AD EDENDVM, NON AEQVE AD BIBENDVM Aegyptiis inferuiebat fuccofa papyrus. Hanc in rem HERODOTVS L.II. in Euterpe affirmat : quod pars papyri inferior, longitudine cubitali in cibos veniat, et venundetur. Omnes certe incolae papyrum fruticem et crudum, et elixum, et affum manducabant, et fuccum deuorabant, manducatnentum autem exfpuebant, ve loquitur GVIL.ANDINVS 1. c. p. 48. Addit ibidem \$.27. teftimonium HERODOTI, quod, fi qui papyro admodum fuauiter frui cupiant, in luculento furno torrefactum ita comedant.

\section{5. $\mathrm{XX}$.}

Vnde facile apparet, quinam fint papyrophagi? Videlicet papyrum, et ex ea panem comedebant Aegyptii, ideoque papyrophagi funt dicti a Scholiafte AESCHILI; fructu papyri et radice etiam vefci folitos fuiffe Aegyptios, licet cibo exigui nutrimenti, narrat Eben-Seraphion fimplicium capite ror. quod tamen quoad fructum negamus, vi Capitis noftrill. S. 17. et 18. Siquidem DIOSCORIDES L. I. C. 125.tan-

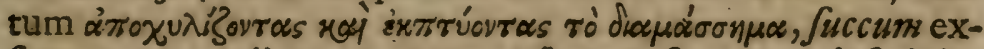
fugentes et reliquum commanfum exfpuentes defcribit, conf. Celeberr. HILLERVS in Hierophyt. fuo P.II.c.XXXVIII. vbi de papyrophagis plura tradit.

\section{XXI.}

Quod vero diximus, papyrum non aeque ad bibendum fuiffe vtilem, id ideo fecimus, vt explicaremus, quid fit vinum biblinum? Supra ftatim Cap. I. \$. I3. obferuauimus, certo

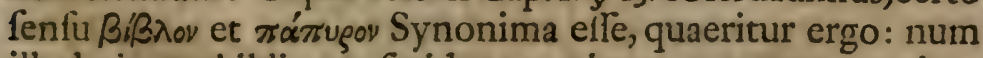
illud vinum biblinum fit idem ac vinum papyraceum, h.e. per torcular ex fungofa et fuccofa fubftantia papyri expreffum? Vini huius mentionem facit HESIODVS L. II. "̈ 5 yov ì

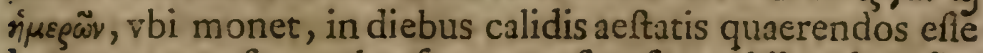
locos cauernofos, vmbrofos atque faxofos, et bibendum oivov BißßAvion h.e. frigiclum. Quod vinum celebrat etiam ACHAEVS 
Tragicus, et THEOCRITVS Idyllio XIV. Sunt, qui vĩnum hocce nomen fortitum effe a Byblo, ciuitate Phoeniciae, aiunt; SVIDAS tum a Byblo ciuitate, tum a Biblia vitis genere dictum putat; GVIL.ANDINVS 1. c. p. 48. vocari vinum biblinum vult inde, quia ins eadem cum Biblo, i. e. papyri regione prouseniat. Nos vero refpondemus, non dari regionem, quae Biblus Aeg ypti audiat, adeoque coniecturam GVILAN D I N I locum inuenire non poffe, practerea papyrus quidem ad edendum et fugendum, aft non vini more ad bibendum erat apta, adeoque, vsque dum aliud edoceamur, vinum biblinum a Biblo, ciuitate Phoeniciae, dictum effe mallemus; nomen vero haec ciuitas adepta fuit a biblo, h. e. papyro A egyptia. Quod fi autem quis cum EVSTATHIO in Iliad. $e^{\prime}$, aut cum ATHENAEO L. 1, Biblinam Thraciae effe regionem, indeque vinum biblinum dictum fuife aftèueret, litem magnam ei non mouebimus, quippe cum Thracia terra fit frigida, et oivov $\beta_{i} \beta \lambda_{\text {w wov a }}$ HESIODO ad refrigerium in calore fit commendatum.

\section{§. XXII.}

V. AD ARMATVRAMI NAVTICAM papyrum quoque fuiffe ab Aegyptiis vfurpatum GVILANDINVS refert, fiquidem varia armamenta nautica inde facta fuiffe, ait, qualia autem ea fuerint, tacet; interim tamen non omni deftituitur fun-

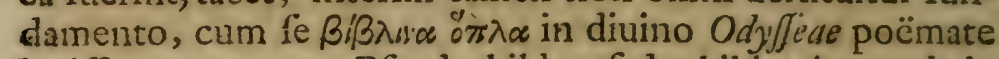
legiffe, eaque non a Pfeudo-biblo, fed a biblo Aegyptia h. e. a papyro nominata ac confecta fuific contra EVSTATHIVM defendat, et adhaec etiam nauigia papyraces fententiam eius confirment.

$$
\text { 5. XXIII. }
$$

VI. AD CALCEOS accommodabatur papyrus, quod bene quoque obferuarunt DALECHAMPIVS ad h. l. Plinii, et CAMP.VITRINGA ad C. XIX.v. 7. Ef. SicHERODOTVS L. II. p.rIG. Sacerdotes quoque calceos papyraceos, úzo-

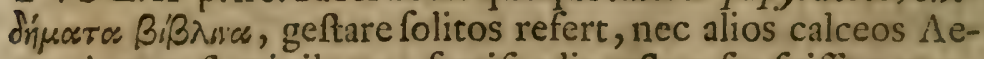
gyptiorum flaminibus ac facrificulis geftare fas fuiffe memorat. SCHEFFERVS vero, vti Summe Reuerendus D. SAL. DEXLINGIVS in Obferu. P. Lul. Difp. de fletu fuper. Tham- 


\section{DE PAPYRO FRV TICE.}

muz p. 524. proea, qua pollet, induftria, adnotauit, $P_{y \text { thago- }}$

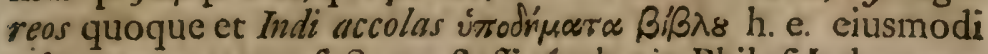
calceos ex papyro factos geftaffe probat in Philof. Ital. p. Isз.

\section{XXIV.}

VII. AD CORONANDOS DEOS quoque papyri vfum hatud exiguum fuiffe facile B. L. intelliget, fi in animum reuocet verba ANACREONTIS poëtae lyrici, fupra ad $\$$. V. huius Capitis allegata,vbi Deum amoris, Cupidinem aduocans, ei videbatur

$$
\begin{aligned}
& \text { : "है? }
\end{aligned}
$$

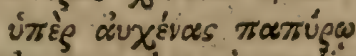

Ђ. e. Cupido quoad tunicam ligatus feu circumdatus papyrs fuper cervice. Procul dubio folia papyri honori coronarum in nuptiis adhibebantur. Hinc PLINIVS fupra 1. c. dixit: nafcitur papyrum femine nullo, aut vfu eius alio, quam floris ad Deos coronandos. Laudat ideo coronam e papyro Agefalaus apud PLVTARCHVM p. 617.

\section{XXV.}

VIII. AD STRATA VARII GENERIS quoque magnum praeftabat vfum papyrus; Hinc PELAGIVS de vitis Sanctorum: vides autem, inquit, Aegyptium veftitum mollibus rebus, et budam a) de papyro, et pellem ftratam fub ipfo. Et paullo poft: pro his dedit mihi Deus framensum de boc papyro, et hanc pellein. b)

a) Buda eft ftramentum vel ftorea, b) Heic vocat framentum de poeine Decke von Binfen-Robr. ISIDORVS budas, ftramenta camelorum i. e. $\sigma \alpha \dot{\gamma} \mu \alpha \tau \alpha$, vel s $\varsigma^{\omega} \mu \alpha \tau \alpha$ alias exponit.

\section{XXVI.} pyro, quam paullo ante budam de papyro vocauerat Eft enim buda ex graeco ßudy', quod a verbo Burw, farcio, impleo, factum eft.

In fpecie legimus papyrum quoque tum ad ceruicalia, tum ad toros mortuorum farciendos valuiffe. «) Ad cer. sicalia quod attinet, rachanas olim de papyro, h. e. fafciculos, qui ceruicalium vice erant, Aegypti habuere, auctore SALMASIO 1. c. p. 706. vbi addit fimul teftimonium ANA. STASII in vita IOANNIS Eleemofynarii, qui fequentem in modum loquitur: illic eos fecerunt, quaje tholos a) quos- 
dam prolixos, ligneis tabulis, pauimento ftrato; ad requiefcendum, et fimul cunt, ftoreis et rachanis per totum biemem. a) tholus eft graecum $\vartheta c i n o 5$, no- coëunt.t. teftudinem ipfam aeditatque t. den Quer-Balcken und um, die Decke, das Gelvoelbe Scblufs-Stein im Gelboelbe, hinc felber, vti h. I. Quam in fentenISIDORVSXIX, 19. tholus pro- tiam VIRGILIVSAen.IX, 408. prie veluti fcutum breue, quod Sufpendiue tbolo, aut facra ad fa. in medio tecto eft, in quo trabes figia fi $i$.

\section{XXVII:}

B) LeCtos autem mortuorum quod fpectat, ad eos farciendos papyrus quoque adhibita fuiffe legitur, quod SALMASIVS ex PLIN. L.XVII.c.37.colligit Verba eius loco citato p.705. ita fluunt: papyro et cirpo, qui naturam babet popyraceam, farciebant lectos murtuorum, (fcilicet Aegyptii,) cum quibus rogo inferebantur ac comburebantur. Vnde Martialis:

Fartus pàpyro dum tibi torus crefcit.

Alimenta igitur ignibus ex papyro et fcirpo quaerebant ad cremanda cadauera.

\section{Tandem quoque}

\section{\$. XXVIII.}

IX. AD VSVM IGNIS, quem papyrus fuppeditauit, nobis deueniendum eft. Non enim folum ad candelas, fed et adcalefaciendum accerfebatur papyri lignum. Candelae quidem e papyro, cera circumdato, parabantur, quibus prae. lucentibus mortui Aegyptiorum noctu poffent efferri. Quam in rem PERSIVS:

Hinc tuba, illinc candelae.

Magis perfpicue modum infuper indicat SALMASIVS 1. c. p. 706. quando fic fatur: detradio cortice, medulla interior candelis faciendis inferuiebat, cera circumdata. Et haec vir excellentis ingenii haufit ex PLINIO, qui L. XVII.c. XXXVII. fqq. e quibus, inquit, detracto cortice, candelae lmminibus et funeribus feruiunt.

\section{XXIX.}

Ad calefaciendum autem Aegyptiis inprimis radices papyri idonei videbantur. Radicibus pro ligno vtebantur, inquit recte CAMP. VI'TKINGA ad locum noftrum, in quo PLI- 
PLINIVS ei praeiuit, qui loco faepe allegato, radicibus, ait, iscolac pro ligno vtuntur, quod mirandum non eft, cum vel noftra charta, papyrus dicta, ad calefaciendum quandoque poffit adhiberi.Sic ignem chartaceum f. papyr oceum a mercatore quodam in honorem Caroli V.accenfum commemorat SCHVZIVS, Paft.Vehlfanzienfis in Appar.Curiof.p.r34r. Quae omnia quo funt 'certiora, et extra dubitationis aleam pofita ; eo minus neceffe erit, ve iis immoremur. Sufficere autem haec nobis videntur de vilitate papyri. Et ne nimislongi fimus, vel tribus adhuc verbis addere volumus

\section{ऽ. XXX.}

III. HISTORTAM OPERARIORVM, MERCATORVRIQVE, quaEtUm et victum ex papyro quaerentium. Quanquam autem ex amplifima vtilitate papyri cuilibet iam iam procliue fit iudicatu, quam multi operarii ex papyro potuerint victum quaerere; iuuat tamen CYRILLI ALEXANDRINI ad locum noftrum Efaianum teftimonium adducere, verba eius To-

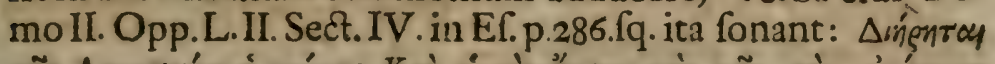

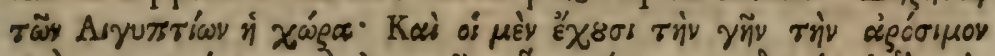
*

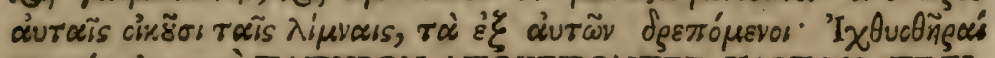

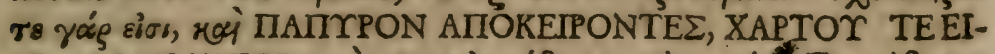

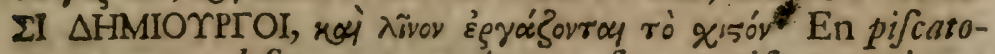
res papyrum defecarmnt, ac chartae fuere artifices, quin et ex chartis, de papyro conficiendis, victum quaefiuerunt.Maiorem lucem nobis accendit MONTFAVCON in Antiqu.Rom. T.III.P. II.L.V.C.5.p.351. verba eius funt: ingens erat multitudo Alexundriae, quae boc diurno-quaeftu (ex papyro facienda) victum pararet, vt indicat HADRIANVS AVGVSTVS in Epiftola fua apud VOPISCVM: Ciuitas opulenta, diues, foecundu, in qua nemo viuat otiofus. Alii vitrum conflant, ab aliis charta conficirur. Vnde apud eundem VOPISCVM legitur : FIRMvi tyrannum tantum babuifje de chartis, vt publice faepe dixerit: exercitum fe alere poffe papyroet glutine; h. e. vt MONTFAVCON cum CASAVBONO explicat: tanta penes FIRMVM tyrannum fuit papyri et glutinis copia, vt is venditis potuerit ex precio exercitum alere; Et 
vere papyri magnum erat per cotum imperium Rom. commercium.

E. $\quad X X X I$.

Et fic denique progredimur ad

\section{CAPVT IV. \\ DE CONSIDERATIONE MORALI \\ ดוา SEV PAPYRI. \\ $\Sigma \Upsilon N O \Psi$ I $\Sigma$.}

Hanc confiderationem vRsINvs, ב omnipotentia Dei $\oint$. XII.

HILLERV', et MONTFAVCON male z bonitas Dei S.XIII.

omijere 8 . $I$.

Oftenditur idcirco necefitas $\oint$. II.

Multi id laudabiliter cognouerunt p. ad Deum colendum f. officie

ex veteribus, et quidem Patri-

bus Graecis et Latizzis 9. Ill. 2. Cole Deum timose ac reuerentia et IV.

Ex recentioribus et recentifinis quoque $\mathrm{g}$. V. et VI.

Confiftit autem confideratio mora-

lis in eo, vt nobis via monftretur

p. ad Deum coginofendum

t. quoad exiftentiam §. VII.VIII.

Idque contra Aibeos S. IX.

Vnde innotefcit eius prouidentia

§. $X$.

x. quoad effentiam f. attributa, qualia funt

s. Sapientia Dei 6. XI.

7 et iufitia Dei, praefertim b. $z$. §. XIV.

Specialia, cuiusmodi funt

f. $X V$.

2 Dei prouidentiae confide S.XVI.

3 Deum tanquam fummum bonum ama S. XVII.

4 Deun celebra laudibus. S. XVIII. $s$ Deo grates agere ne obliwifcere 6. Xix.

6 Colc Deum ei obediendo ac feruiendo. S. XX.

Quae omria Aegyptii non obferuarunt, a Deo inde puniti. Et fic additur claufula. \$. XXI.

5. 1.

A Yordimur hoc Capite eam tractationem, quam moralem 1. nfcripfimus, quaeque nobis officia,ex contemplatione Tiny Acgyptiis piopria exponit. Miratus fum, quod VR. SINVS in Aiboreto fuo l.c. qui alioqui praecepta moralia זractationi arborum eleganter infpergit, idque cum eo HILLERVS in laudato Hierophytico fuo I. c. nec non Celeberr. MONTF $\triangle$ VCON, tam multa l.c. de papyro afferens, hoc omiferint. Maximam enim partem confiderationis abfoluit enucleatio moralitatis, inde etiam vfus ex contemplatione in nos redundat. §.II. 


\section{DE PAPYRO FR\&TICE.}

\section{S. II.}

Adeoque fummae eft necefitatis, cuilibet plantae, cuilibet arbori, cuilibet herbae, quam in hac mundi machina confiderare coepimus, adnectere confiderationem moralem, praefertim vbi planta biblica enarratur. Eruditi eft inde elicere fines Dei, hominis gentilis inde elicere Theologiam naturalem, Chriftiani autem inde deducere regulas vitae ad fanctitatis incrementum.

\section{III.}

Multi laudabiliter id cognouerunt, praelucente S. Scriptura, maxime diuino $P$ falmifta, iique tum veteres, tum recentiores.

\section{ઈ. IV.}

Ex veteribus proftant Patres Graeci a) aeque ac Latini, b) qui dum Hexaëmeron Dei defcripferunt, in genere quandoque etiam nonnihil moralitatis confiderationi plantarum immifcuerunt.

a) Huc pertinet BASILIVS, de cuius Editt. et Verfionn. legendus eft Celeberr. FABRICIVS in delectu argumentorum pro veritate religion. p. 48. fq. EVSTATHIVS Antiochenus, quem ALLA TIVS in Latinum tranflulit, notisque eruditis illufrauit, de quo videatur eiusd. FABRICII Biblioth. Graec. p. 169. His addimus SEVERIANI, Gabalorim in Syria Epifcopi Homilias VI. in Cojmopoeiam, quae extant in longe elegantiffima Editione BERN. de MONTFAVCON T. VI.

$$
\text { \$. V. }
$$

Ex recentioribus in fpecie id fecerunt 'Theologi aeque ac Philofophi Chriftiani, inprimis Angli. a)

a) Adducamus hic faltem notatu dignifimos, quales funt: IO. GERSON, Cancellarius Parif: qui edidit Centilogium de sansa p. 437. cum Verfione COMBEFISII.

b) Latinis annumeramus AMBRUSIVM in Libb. VI. Hexacmer. in quo quidem Opere vbiqueBASILII premit veftigia. Huc referri poffunt et alii, qui ad modum Patrum de Opere 6. dierum feripferunt, vt BEDA Venerabilis in Hexaëmer. T.IV. Opp. Huic a latere eft ponendis IOIPICVS MIRANDVLANVS in Cpere fex dierum. IO. BLOMIVS in Hexaëmero Hamburg. 1664. edito. 
T. I. Opp. p. 676. ROBERTVS BOYLE in' $T$ r. quem infcripfit: of the high veneration man's intellect owes to God, peculiar ly for bis twifedom and po: wer, Lond. 1685. 8. vbi nos ad Sapientiam Dei vel ex plantis elucentem ducit. SAM: PARKER in Cogitationibus de Deoet prouidentia, Lond. $16 g 6$. Porro §. VI.

Ex recentiffinis in hoc campo illuftres fefe dederunt CHRIST.WOLFF.a) BERN.NIENWENTYT,b) WILLIAM DERHAM, c) et cum iis IO. GEORG.WALCH. d) a) p. in Ab/icbten der vatiirlichen c) In praeftantiffino Tr. cui tituDinge Hal. 1723. 8. p. im Gebrauch der Theile am Menfchen, Thiere und Pflantzen ib. 1725.8.

b) In eleganti Opere, quod inferipit: Het regt gebruik der Werelt befcholoingen, ter overtuiginge van Ongelovigen, Amftel. 1715.4. Huic adiungendus eft RAIVS in Wifedom off $G \otimes d$, qui iam ante eruditis innotuit biftoria plantarum de quo fupra adCap. III. 6 . I. diximus.

lum dedit: Pbyfico - Theology. HuiusVerfionem Germanicam procurauit Celeberr. IO. ALB. FABRICIVS Hamb. riz 32 . cum ante viderit Italam, Hollandicam, Gallicamque Verfionem, conf. Acta Erudit. A. 1716.p.106. et Unfchuldige Nachrichten A. 1716. P. 283.

d) In Lex. Philofoph. fub titulo: vonden Pflantzen p. m. 1965.
NATHAN CHYTRAEVS in

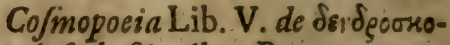
Tía f. de ftirpibus, Brem. I593. 8. vbi cognitio Phy fica ad Dei prognofcendam applicatur. Et $\mathrm{B}$. MOREAU in Tr. qui infcribitur: Confiderations morales tirees des ouvrages de la Nature et de $[$ Art, a Liege 1682. fol. uidentiam et potentiam inde co-

\section{S. VII.} nobis via inde monftretur ad Deum tum cognofcendum, tum culcndum, тraider yoyia nempe naturali. Deum fi inde cognofcere velimus, illiusmodi cognitio referenda eft partim ad eius exiftentian, p. ad eius effentiam f. attributa.

\$. VIII.

Quod ad exiftentiam attinet, papyrus in Aegypto nafcens, nos condocet exiftere Deum. Nam ratio cognofcit ex effectu caufam, ex opere auctorem, ex fine fapientiffimo conditorem. Collige: papyrus frutex non eft a fe ipfo, fed abalio, vim crefcendi indente; Si ab alio, is perfectiffimus, fapientiffimus potentisfimusque fit neceffum eft; fiquidem planta illa, papyrus, quoad minimas partes eft perfectisfima, quoad vfum fapientisfi- 


\section{DE PAPYRO FRVTICE.}

pientifime producta, et quoad primam productionem, continuainque fui multiplicationem potentifine procreata. Sic videbis dari ens perfectislimum, fapientisfimum, potentiflumum, quod eft Dcus. Sane

Praefentem refert quaelibet berba Deum.

6.IX.Vnde Atheifmum plane cum ratione et experientia pugnare, immo “" $\lambda$ oyov effe quilibet videt, et recte laudatus WILLIAM DERHAM L. c. L. XI. c. III. oltendit. conf. PS. XIV, I.

6.X.Ratio deprehendit porro, ens hocce perfectifinum,quod Deun vocamus, non tantum papyri effe caufam, fed per eamdem etiam prouida fua cura Aegyptiis profpexiffe, eorumdemque alimenta, veftes, incrementa hoc modo procuraffe, ex quo Dei prouidentia fatis fuperque clucet.Quid enim tam furiofum effet, quamDeum creatorem non negare, gubernatorenn negare, dicere eum factoremn efé omnium, eumdem vero. quae fecit negligere, inquit, SALVIANVS de gubernat. Dei L. VII.p. 262. et IO.LASSENIVS in beiligen Moralicn p. 535.Plura hanc in rem edifferit IO. FRANC. BVDDEVS int thefiluss de atbeifmo et fuperfit. ad C. I, 5. 29. p. 69. item ad c.III. \$. I. p. 24 1.fq.

5.XI.Quoad efentiam f.attributa aliquo modo etiam ex papyroDeus goteft cognofci. Nos potiora, quae huc fpectant, breuifimis adponemus. Sapientia Dei omnium primo Aegyptiorum poterat fuccurrere menti. Quodfi enim omnes partes huius fruticis, quas Cap.II.fupra defcripfimus; fi fitum et natales eius על עיפי יאור vbi fuccì, adeoque vitae defectus non erat extimefcendus, animo fecum volutarunt, non potuerunt non inde admirari Numen Sapientiflunum, quod omnia tam artificiofe, tanque follicite ad finen certum creauit. Vnde merito Dauid exclamat: quam magna funt opera tua, o Deus, fundae funt cogitationes thac! Pf. CIV, 24. et PAVLVS: o quantae profunditates fapientiae Dei funt! Rom. XI, 33.

6.XII. Alterum attributum ex papyro elucefcens, eft infinita Dci potentia, fapientiac eius confobrina.Etiamfi enim Deus de creanda ciusmodi planta, quaedegyptiis proficua foret, cogitaflet; nifi potentia eius immenfa fuiftet, fruftra fuiffet fapientia. O quanta ex aduerfo ent imputentia hominum, qui ne folium quidem minutifimae herbae fua virtute, fuo marte exterra proferre valent!

§.XIII.Tertium attributum Dei efto bonitas eius. Reputet quaefo, fecum L. B.ea, quae Cap. antec.de vfu papyridiximus; reputet quaefo, recum, quod Deus mon alibi tam copiofe, et vtiliter progerminare paftus fit fapyrum, et inde mirabilia fane bonitatis div. argumenta A egypt iis fere approbaffe animaduertet. Sed abundantia papyri ingratis incolis Aegypti excitabat faftidiun, et iuftam proinde Dei largitoris Benigniffimi iram, cum qua nos ducimur ad

6. XIV. Iuftitiam Dei tanquam quartum attributum cius. Certe quain clemens Deus eft, tam iuftus ac terribilis $i_{i}$ a elt! Dictum noftrum Efaianum de eo fatis nos ̧ouvincit. 


\section{DE PAPYRO FRVTICE.}

§.XV.Ecce hoc modo papyrus $\Lambda$ egyptios ad cognofcendum Deum; cum quoad exiftentiam,tum quoad effentiam f attributa potuit inflam. mare. Sed praeterea cosdem etiam ad colendum Deum r. ad officia fpecialia, religioni naturali inferuientia, potuit manuducere. Ninirum vbi adeft cognitio Dei, papyri autoris, fapientis, potentis ac iufti, ibi debet adeffe timor ac reuerentia. Officium primum itaque, ex doctrina de papyro refultans, eft : cole Deum timore ac reuerentia.

S.XVI. Cumque Deus eorum faluti per papyrum profpexerit oculs fuo prouido, quem ipfimet Hieroglyphice olim depinxere, debebant ve-

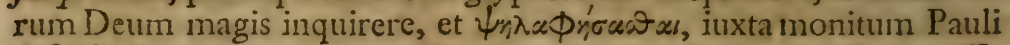
Act.XVI1,27. eique tanquam fummo rerum moderatori, et benigniflmo papyri conferuatori confidere, et inde alterum officium erit: Dci prousidenticie conffde:

6.XVII. Haec, fi quis penitus introf piciat, Deum papyri, fruticis tam inauditae vtilitatis largitorem, non folum admirabitur, fed ct amore dignum iudicabit, tanquam fuper omnia pulchrum, clementem et ama. bilem : tertium itaque officium praecipit: D:um tanquam fummum bonum ama.

6. XVIIII. Quod amore dignum eft, illud promeretur procul dubio laudem. Quartum igitur officium eft: lauda Deum pafyri creatorem. No n defatigemur in laudando Deo,cum non fuerit laffus in procreandis tam ftupendis operibus, praeclare loquitur GALEN. de vfu part. L. VII. c. 18. Idem monet DERHAM in Phy fico-Theologiae L. XI. c. 2. Recte Dauid laudat Deum ob arbores, quae ab co reve Saturantur fe. humore, cuius Dauidis veftigia fequamur.

9.XIX. Quoties Aegyptii manducabant, tractabant, vfurpabant papy$r_{u} \mathrm{~m}$, fibi in vfum a Den conceffan, toties etiam ad $\chi \alpha \rho, \tau$ chcjías et grain arum actionem poterant ac debebant inuitari. Vnde noumm emergit officium illud: Deo gratis agere ne obliuifcere.

f.XX. Denique etiam Deus iure creationis et dominii merito ab Aegyptiis, papyri quafi dominis a fe conflitutis, poftulauit, vt ei obedirent atque probe ponderarent, fe aliquando ad rationem de abufu papyri vocatum iri, fextum ergo officium mandat: cole Detum ci obediendo ac forticisdo. Voluntatem Dei, Domini fui, nouerant Aegyptii, tum per legem cordibus omnium inferiptam Rom. II, 15. tum per Prophetas, vt. h. l. per Efaiam, annunciatam. Vnde illo dic apparebit,qui Deo feruituerit, quique non feruiuerit Mal. IJI, I8.

§. XXI. Quae omnia tamen repudiauit Aegyptus, eitusque incolae, natura lem de Deo reprimentes notitiam, eligentes idola, quare Deus hoc Cap. XIX. Efa. v.I-7. dicitur eos puniturus exficcatione Nili et papyri, quot etiam factum eft. Felix, quem faciunt aliena pericula cautun! Et fic Deo agimus grates, quod adeft finis.

$$
\text { C O R O L L A R I A: }
$$

1. Theologia naturalis eft per modum babitus connati infita. II. Dantur plantue fine femine, vti noftra papyrus. 


\section{I R I S}

EXCELLENTISSIMO A'TQVE AMPLISSIMO

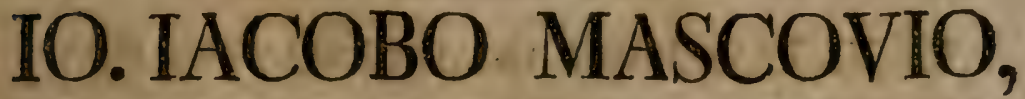

IVRIS V'TRIVSQVE DOC'TORI, IN SVPREMA CVRIA PROVINCIALI PARITER A TQVE CONSISTORIO ADSESSORI, IVRISQVE PROFESSORI PVBLICO, CAPITVLI CIZENSIS CANONICU, INCLVTAE REIPVBLICAE LIPSIENSIS SENATORI,COLLEGIIQVE MINORIS PRINCIP. COLLEGIATO, NEC NON

MAXIME REVERENDO AMPLISSIMOQVE

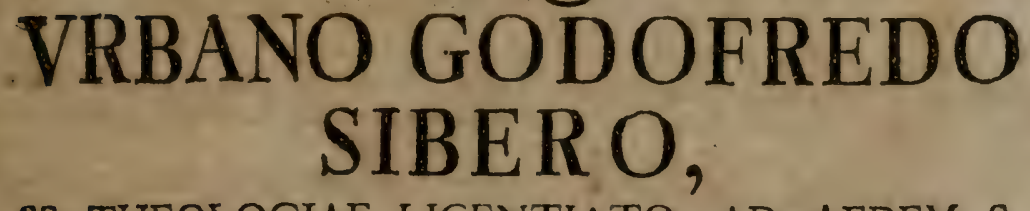

SS. THEOLOGIAE LICENTIATO, AD AEDEM S. THOMAE LIPSIENSIVM ARCHI-DIACONO, ANTIRVITATVM ECCLESIASTICARVM PROFESSORI PVBI.ICO.

PATRONIS AC MAECENATIBVS SVIS OP'TVMIS, MAXVMIS 
HAS PAGELLAS

DEVO'TA MEN'TE

AD VLTERIOREM COMMENDA'TIONEM

\author{
AC PA'TROCINIVM, \\ VETERI CLIEN'TVM MORE \\ D. D. C.
}

\title{
DEVOTISSIMVS CLIENS \\ M. 10. GODOFREDVS VNGERVS, \\ SEMITAR, PAVLL, EACVLTAT. THEOL, LLPS. MEMBR.
}




Gaylord Bros,

Makers

Syracuse. N. Y,

PAT. JAK. 21, 1808 
in: 